\title{
The Protective Effects of the Autophagic and Lysosomal Machinery in Vascular and Valvular Calcification: A Systematic Review
}

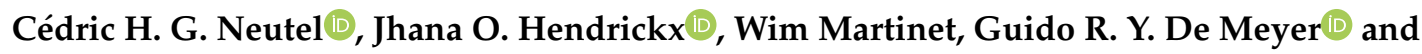 \\ Pieter-Jan Guns *(D) \\ Laboratory of Physiopharmacology, University of Antwerp, 2610 Antwerp, Belgium; \\ cedric.neutel@uantwerpen.be (C.H.G.N.); jhana.hendrickx@uantwerpen.be (J.O.H.); \\ wim.martinet@uantwerpen.be (W.M.); guido.demeyer@uantwerpen.be (G.R.Y.D.M.) \\ * Correspondence: pieter-jan.guns@uantwerpen.be
}

Received: 5 November 2020; Accepted: 21 November 2020; Published: 25 November 2020

check for updates

\begin{abstract}
Background: Autophagy is a highly conserved catabolic homeostatic process, crucial for cell survival. It has been shown that autophagy can modulate different cardiovascular pathologies, including vascular calcification (VCN). Objective: To assess how modulation of autophagy, either through induction or inhibition, affects vascular and valvular calcification and to determine the therapeutic applicability of inducing autophagy. Data sources: A systematic review of English language articles using MEDLINE/PubMed, Web of Science (WoS) and the Cochrane library. The search terms included autophagy, autolysosome, mitophagy, endoplasmic reticulum (ER)-phagy, lysosomal, calcification and calcinosis. Study characteristics: Thirty-seven articles were selected based on pre-defined eligibility criteria. Thirty-three studies (89\%) studied vascular smooth muscle cell (VSMC) calcification of which $27(82 \%)$ studies investigated autophagy and six $(18 \%)$ studies lysosomal function in VCN. Four studies $(11 \%)$ studied aortic valve calcification (AVCN). Thirty-four studies were published in the time period 2015-2020 (92\%). Conclusion: There is compelling evidence that both autophagy and lysosomal function are critical regulators of $\mathrm{VCN}$, which opens new perspectives for treatment strategies. However, there are still challenges to overcome, such as the development of more selective pharmacological agents and standardization of methods to measure autophagic flux.
\end{abstract}

Keywords: vascular calcification; aortic valve calcification; autophagy; lysosomes; vascular smooth muscle cell (VSMC); valvular interstitial cell (VIC)

\section{Introduction}

Autophagy ("self-eating") is a highly conserved catabolic process, responsible for the degradation of long-lived cytosolic proteins and organelles [1]. Three distinct forms of autophagy have been reported in mammalian cells: Macroautophagy, microautophagy and chaperone-mediated autophagy (CMA). While all three forms result in the delivery of cargo to the lysosomes for degradation, macroautophagy (hereafter referred to as autophagy) is the most investigated form [2,3]. The autophagic machinery is finely regulated, starting with generating an isolation membrane, known as the phagophore (Figure 1). The phagophore expands and engulfs intra-cellular cargo, resulting in the formation of a double-membraned structure, called the autophagosome. These autophagosomes fuse with lysosomes, forming autolysosomes that eventually degrade the autophagosomal cargo [3]. Consequentially, lysosomal function is closely entangled with autophagy function, as defective lysosomal activity impairs autophagic flux [4]. Additionally, autophagy can be specific in selecting its cargo for degradation. Pexophagy (peroxisomes), mitophagy (mitochondria), lipophagy (liposomes), ribophagy (ribosomes), 
xenophagy (intracellular pathogens) and aggrephagy (aggregated proteins) are examples of selective autophagy, mediated by specific autophagy receptors [5].

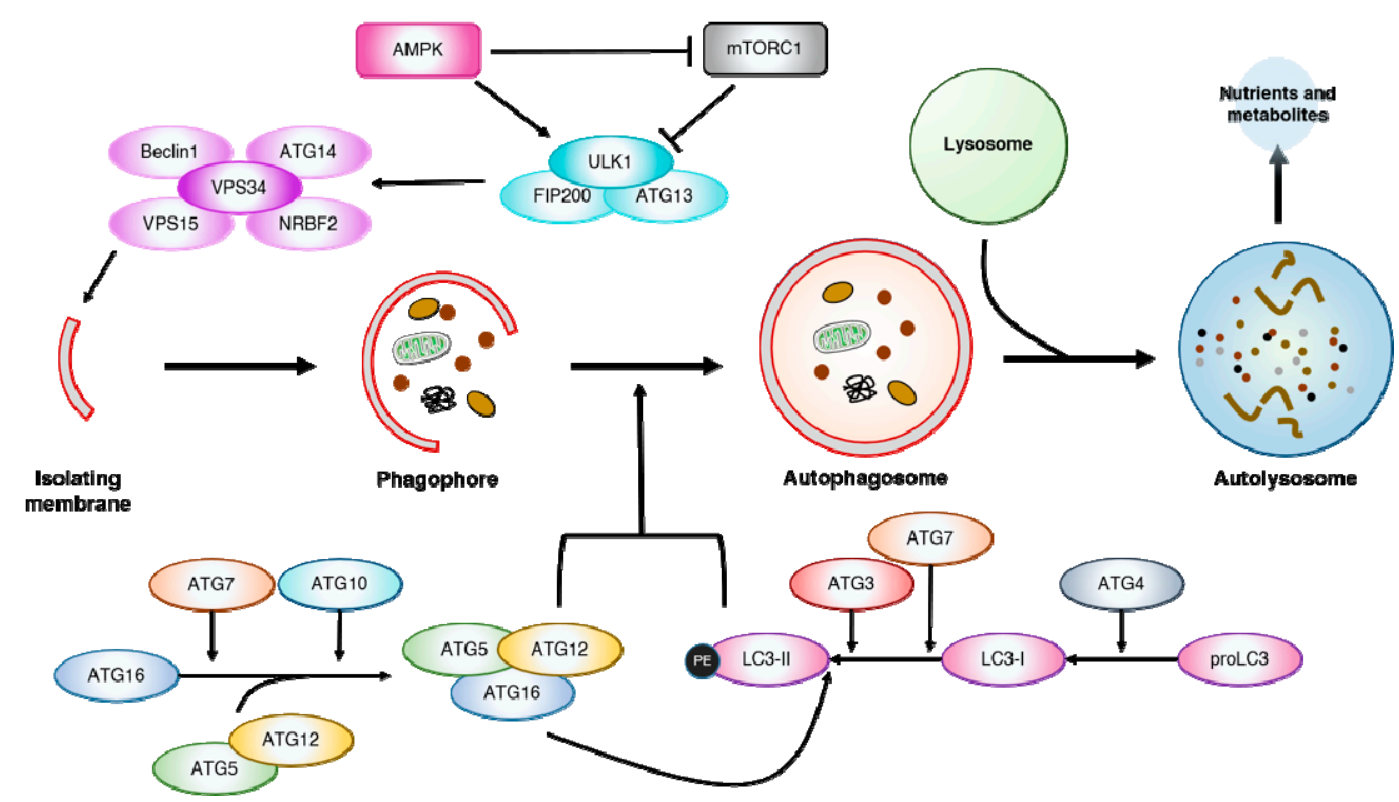

Figure 1. The autophagic process. The autophagic machinery starts with the formation of an isolation membrane, forming the double-membraned intermediate, the phagophore. The maturation of the phagophore into an autophagosome is dependent on the activity of two ubiquitin-like conjugation systems, the LC3-PE and Atg5-Atg12. In the first system, pro-microtubule-associated protein light chain 3 (proLC3) is cleaved by the cysteine protease Atg4 into LC3-I. LC3-I is then further processed into LC3-II and conjugated with phosphatidylethanolamine (PE) by Atg7 and Atg3, which are E1- and E2-homologous enzymes, respectively. LC3-II associates with the autophagic membranes with the aid of the Atg5-Atg12-Atg16 complex, facilitating phagophore maturation into an autophagosome. The Atg5-Atg12-Atg16 complex is formed by the action of Atg7 and Atg10, E1 and E2 enzymes respectively. This newly formed autophagosome then fuses with a lysosome forming an autolysosome. Here, lysosomal enzymes are responsible for the degradation of the autophagosome and its contents. Once degraded, the macromolecules (nutrients and metabolites) are secreted back into the cytosol. The shapes inside the phagophore and autophagosome are intra-cellular cargo such as (dysfunctional) mitochondria, (aggregated) proteins, etc. targeted for degradation; Atg = Autophagy related proteins.

Multiple autophagy-related genes (ATG) have been identified, whose products are specific to the autophagic process [6]. Autophagy is regulated by these Atg proteins and mediated by upstream signalling, with the mechanistic target of rapamycin (mTOR) and AMP-activated kinase (AMPK) as the most important regulators. In short, mTOR represses autophagy induction by inhibiting unc-51 like autophagy activating kinase 1/2 (ULK1/2) and the vacuolar protein sorting 34 (VPS34) complex, while also preventing global expression of lysosomal and autophagy genes via phosphorylating transcription factor EB (TFEB) [7]. In contrast, AMPK, a cellular energy sensor, induces autophagy in low cellular energy conditions by both inactivating mTORC1 and phosphorylating ULK1 [8]. Additionally, AMPK induces autophagic flux by modulating both autophagosome maturation and autolysosome fusion [9]. The formation of autophagosomes relies on two ubiquitin-like conjugation systems: (1) microtubule-associated protein light chain 3 (LC3)-phosphatidylethanolamine (PE) (LC3 is the mammalian homolog of yeast Atg8) and (2) Atg5-Atg12 that have an essential role in autophagy and are widely conserved among eukaryotes $[6,10]$. In the first system, proLC3 is cleaved by the cysteine protease Atg4, forming LC3-I [11]. LC3-I is then conjugated with PE by Atg7 and Atg3 which are E1and E2-homologous enzymes respectively, forming LC3-II that associates with autophagic membranes and is often used as a marker for autophagy [10,11]. In the second system, Atg5 is linked to Atg12 by 
the E1 and E2 enzymes Atg7 and Atg10, which further establishes a complex with Atg16L1 [11,12]. These Atg5-Atg12-Atg16L1 complexes oligomerize, forming larger units, which are required for targeting LC3 to the autophagic membranes [10]. These two conjugation systems are interdependent since LC3 conjugation is dependent on the activity of the Atg5-Atg12-Atg16L1 complex [10].

Autophagy has a central role in maintaining cellular homeostasis by eliminating unwanted, harmful cytosolic material [12]. The autophagic elimination of dysfunctional mitochondria or protein aggregates is necessary for mitigating the release of pro-apoptotic mediators and reactive oxygen species, safeguarding cell survival [11,13]. Accordingly, autophagy modulates many pathologies, playing an important role in cardiovascular diseases such as atherosclerosis, heart failure, arrhythmia, chemotherapy-induced cardiotoxicity, as well as being implicated in cardiac and vascular aging [14-16]. More recently, autophagy has been found to protect against vascular medial calcification, which is considered as an active, pathological and multifactorial phenomenon that is distinct from atherosclerosis [17-22]. Cardiovascular calcification features the deposition of calcium phosphate minerals, mainly hydroxyapatite $\left[\mathrm{Ca}_{10}\left(\mathrm{PO}_{4}\right) 6(\mathrm{OH})_{2}\right]$ crystals, in cardiovascular tissues including arteries, heart valves, and the cardiac muscle [23-25]. Recent research showed that vascular smooth muscle cells (VSMCs) in the media can differentiate to osteoblast-like cells and form matrix depositions, making the process of vascular calcification ( $\mathrm{VCN}$ ) very similar to bone ossification [21,25-28]. VSMCs undergo osteogenic differentiation driven by increased intracellular concentrations of calcium and/or inorganic phosphate $(\mathrm{Pi})[23,26,29]$. An indispensable transcriptional regulator for this osteoblastic differentiation is the core-binding factor subunit $1 \alpha /$ runt related transcription factor, Cfba1/Runx2, causing VSMCs to release small phospholipid-bound matrix vesicles (MVs) [22,23,29-31]. Released MVs contain alkaline phosphatase and annexins that promote the deposition of hydroxyapatite crystals. Moreover, the Cfba- 1 transcription regulator controls the secretion of bone-associated proteins, such as osteopontin, collagen type 1, osteoprotegerin and osteocalcin [23,26,32-34]. Besides numerous transcriptional and pro-osteogenic factors, bone morphogenetic proteins (BMPs) are upregulated within calcifying VSMCs [28,35]. The latter protein family consists of 15 members and forms a sub-group of the TGF- $\beta$ super-family. These proteins play an important role in VCN and bone proliferation, development and fracture healing [36-38]. Within the BMP protein family, BMP2, BMP7 and BMP9 have been reported to have the greatest osteogenic-promoting activity [35,39-42]. BMP2 increases the uptake of Pi, promotes RANKL osteoblastic activity in VSMCs and induces VCN via the Wnt/ $\beta$ catenin pathway $[38,43-46]$.

Today, therapeutic options for treating VCN are limited. Targeting the autophagic and/or lysosomal machinery has been proposed as a promising strategy for drug development in VCN. This review aims to gather the current knowledge regarding the benefits and drawbacks of modulating autophagic-lysosomal function in VCN and aortic valve calcification (AVCN). Therefore, studies with pharmacological agents that modulate the autophagic-lysosomal machinery in VCN are listed. A recent review summarized the underlying mechanisms by which autophagy protects from VCN [47]. They broadly discussed how the protective effects of autophagy on VCN are linked with apoptosis, osteogenic differentiation and the release of MVs. However, they did not provide a systematic overview of how modulating autophagy, with existing pharmacological agents, affects VCN.

\section{Objectives}

Lately, autophagy has gained a lot of interest in the VCN research field, with emerging evidence suggesting that autophagy plays a protective role by inhibiting apoptosis and MV secretion [48,49]. Different research groups are exploring autophagy as a treatment against VCN. Therefore, new and exciting data become available, that improve our understanding of autophagy and its possible pharmacological modulation in VCN. This review aims to provide a comprehensive overview of the current knowledge regarding the modulation of the autophagy-lysosomal pathway in both VCN and AVCN. Different interventions that either promote or inhibit the progression of VCN are compared 
and the underlying molecular targets are discussed. Additionally, this review will critically discuss the applied methods for evaluating autophagic activity.

\section{Methods}

\subsection{Search Strategy}

The PRISMA guidelines were followed to assess the literature regarding the role of autophagy and lysosomal function in VCN [50]. The databases MEDLINE (PubMed Database), the Cochrane Library and the Web of Science were consulted up to 29 June 2020. The search string for the databases was: [\{(Autophag*) OR (Autolysosome) OR (Mitophagy) OR (ER Phagy) OR (lysosomal)\} AND \{(Calcif*) OR (Calcinos*)\} NOT (Atheroscler*)].

\subsection{Inclusion and Exclusion Criteria}

Studies were included if they investigated mechanisms of autophagy and/or lysosomal function in VCN, either through genetic alterations or (non-)pharmacological intervention. Both in vivo and ex vivo/in vitro animal and human studies were included. In vitro studies using cells other than vascular and valvular cells (VSMCs, valvular interstitial cells (VICs)), were excluded, as well as studies investigating other forms of pathological mineralization. The publication date was not an exclusion criterion. The reference lists of the included articles were checked to identify additional relevant studies. Only primary research articles were used and therefore abstracts and reviews were excluded. Furthermore, only English language articles were included.

\subsection{Data Extraction}

For the included articles, the type of modulation, the methods, the molecular findings and the study conclusion were extracted. The types of modulation regarding the autophagy-lysosomal pathway are divided in three categories, namely: (1) (non)-pharmacological modulations, (2) genetic modulations, and (3) no intervention/modulation (=observational).

\section{Results}

\subsection{Study Selection}

The search strategy resulted in a combined number of 602 articles, extracted from all three databases (Figure 2). After removing all duplicates, 457 articles were manually checked for relevance by screening their titles and abstracts. Forty-six articles were selected, of which 10 were excluded after full text review. Through the review of the reference lists of the included articles, an additional reference was found eligible and was included. Hence, 37 articles were included for this systematic review.

\subsection{Study Characteristics}

The characteristics of the included studies are presented in Tables 1 and 2. Most of the studies were published in the time period 2015-2020 (92\%), with 58\% of the studies published between 2019 and 2020. Regarding the studied matrix, 32 studies (89\%) studied aortic/VSMC calcification while four studies $(11 \%)$ studied valvular calcification. Among the studies investigating aortic/VSMC calcification, $26(82 \%)$ studies investigated the autophagic machinery in calcification, while six $(18 \%)$ studies explored lysosomal function in VCN. In total, seventeen studies conducted both in vitro and in vivo research, while sixteen studies solely conducted in vitro research and two studies solely in vivo research. Three studies conducted ex vivo research with tissue from human patients. Overall, most studies used different pharmacological and/or genetic approaches to study the effects of modulating autophagy or lysosomal function in VCN. Therefore, together with the significant heterogeneity regarding methods and output analysis, it was not possible to perform a meta-analysis on these data. 


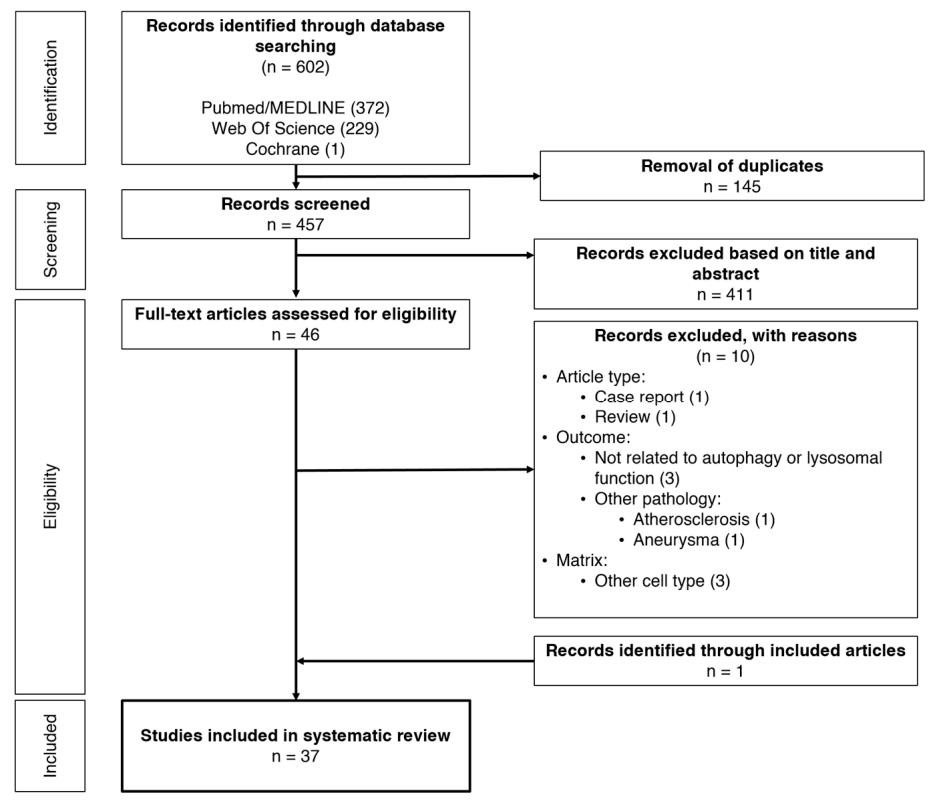

Figure 2. Review workflow.

\subsection{Autophagy in Vascular Calcification}

The studies included in the current review focused, either on modulating (induction or inhibition) autophagy in calcifying conditions (i.e., high Pi and/or high calcium), or on describing how a pro-calcific environment affects autophagic flux or a combination of both (Table 1). Induction of autophagy was established through mTOR inhibition, AMPK activation or unspecified mechanisms stimulating autophagic flux. Additionally, different studies used autophagy inhibitors, either by itself or in combination with an autophagy inducer to demonstrate the involvement of autophagy.

\subsubsection{The Effect of Vascular Calcification on Autophagic Activity}

Autophagic flux was upregulated in VCN in both a chronic in vivo renal failure rat model, as well as in an in vitro Pi-induced VSMC calcification assay [51]. This observation was also confirmed in other studies, where autophagy was upregulated in in vitro and in vivo models of VCN $[52,53]$. Conversely, there is also evidence that mTOR (an inhibitor of autophagy) is upregulated in VSMC calcification [54]. In line with these findings, epigenetic regulation of mTOR activity was also altered in VCN. Histone deacetylase 1 (HDAC1) was downregulated in VCN while lysine-specific histone demethylase A1 (LSD1) was upregulated, resulting in enhanced mTOR activity [52]. Lastly, hydroxyapatite was shown to cause cell injury, leading to decreased lysosomal integrity and facilitating VCN [55].

\subsubsection{Stimulating Autophagy in Vascular Calcification through mTOR Inhibition}

Inhibition of mTOR with rapamycin, adiponectin, bavachin or changing epigenetic regulation of mTOR (through HDAC1 and LSD1) consistently ameliorated VCN. Rapamycin and adiponectin, two known mTOR inhibitors, strongly attenuated VSMC calcification in vitro [54]. In line with these findings, mTOR overexpression aggravated VCN, whereas kinase-dead mTOR expression diminished VCN [56]. (Over)expression of mTOR supressed Klotho expression, whereas rapamycin rescued this suppression and alleviated VCN. Since Klotho knockout mice developed aggravated VCN, it was proposed that mTOR promotes VCN through suppression of Klotho [56]. Furthermore, the flavonoid bavachin ameliorated VCN by inhibiting mTOR, whereas wortmannin or Atg7 knockdown abrogated the protective effects of bavachin in vitro [57]. Lastly, HDAC1 was downregulated in VCN, while LSD1 was upregulated. HDAC1 overexpression or LSD1 silencing resulted in less VCN, mTOR inhibition and autophagy induction [52]. 
Table 1. Studies on the modulation of autophagy and lysosomal function in vascular calcification.

\begin{tabular}{|c|c|c|c|c|}
\hline Authors & $\begin{array}{c}\text { Type of } \\
\text { Intervention/Modulation }\end{array}$ & Methods/Matrix & Molecular Outcome & Study Conclusion \\
\hline \multicolumn{5}{|c|}{ Modulation of the autophagic machinery in vascular calcification } \\
\hline $\begin{array}{c}\text { [51] } \\
\text { (Dai et al., 2013b) }\end{array}$ & $\begin{array}{c}\text { Non-pharmacological: } \\
\text { Adenine diet-fed rats (CRF rats) } \\
\text { Pharmacological: } \\
\text { Autophagy inducers/inhibitors }\end{array}$ & $\begin{array}{l}\text { In vivo } \\
\text { Wistar rats-CRF rat model } \\
-\quad \text { Adenine }(0.75 \%) \text {-induced CRF } \\
\text { In vitro } \\
\text { Primary rat VSMCs and BASMCs } \\
\text { Rat aortic ring calcification } \\
-\quad \mathrm{CaCl}_{2}(1.8 \mathrm{mM})+\mathrm{Pi}(3 \mathrm{mM}) \\
\quad \text { induced calcification }\end{array}$ & $\begin{array}{l}\text { CRF/high Pi: } \uparrow \text { Autophagic flux; } \uparrow \text { LC3-II, } \uparrow \text { VSMC } \\
\text { calcification; } \uparrow \text { MV release } \\
\text { 3-MA: } \uparrow \uparrow V S M C \text { calcification; } \uparrow \uparrow M V \text { release } \\
\text { Atg5 siRNA (in vitro): } \uparrow \uparrow V S M C \text { calcification } \\
\text { Valproic acid: } \downarrow \text { VSMC calcification }\end{array}$ & $\begin{array}{l}\text { Autophagy is upregulated in } \mathrm{VCN} \text { and } \\
\text { counteracts its progression, while } \\
\text { autophagy inhibition has } \\
\text { pro-calcification properties }\end{array}$ \\
\hline $\begin{array}{c}{[52]} \\
\text { (Zhou et al., 2020) }\end{array}$ & $\begin{array}{c}\text { Genetic: } \\
\text { Histone deacetylase HDAC1 } \\
\text { overexpression }\end{array}$ & 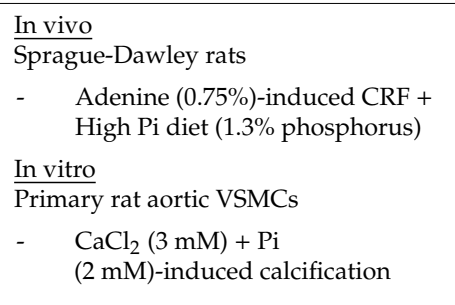 & $\begin{array}{l}\text { CRF rats/High Pi: } \uparrow \text { Calcification, } \downarrow \text { HDAC1, } \\
\uparrow \text { LC3-II, } \downarrow \text { p62, } \uparrow \text { LSD1 } \\
\text { HDAC1 overexpression: } \downarrow \text { Calcification, } \downarrow \text { Runx2, } \\
\uparrow \alpha-S M A, \uparrow \text { LC3-II, } \downarrow \text { p62, } \downarrow \text { LSD1 } \\
\text { LSD1 silencing: } \downarrow \text { Calcification, } \\
\downarrow \text { (p-p70S6K/p70S6K, p-rpS6/rpS6), } \text { SESN2, } \\
\uparrow \text { LC3-II, } \downarrow \text { p62 } \\
3 \text {-MA: } \uparrow \text { Calcification, } \uparrow \text { Runx2, } \downarrow \alpha-S M A \\
\text { Valproic acid: } \downarrow \text { Calcification, } \downarrow \text { Runx2, } \uparrow \alpha \text {-SMA }\end{array}$ & $\begin{array}{c}\text { HDAC1 overexpression attenuates VCN } \\
\text { by inhibiting LSD1 via SESN2-dependent } \\
\text { mTOR signalling }\end{array}$ \\
\hline $\begin{array}{c}{[53]} \\
\text { (Frauscher et al., 2018) }\end{array}$ & $\begin{array}{l}\text { Pharmacological: } \\
\text { Rapamycin }\end{array}$ & $\begin{array}{l}\frac{\text { In vivo }}{\mathrm{DBA} / 2 \mathrm{NCrl} \text { mice }} \\
-\quad \begin{array}{l}\text { High Pi diet (HPD; } 20.2 \mathrm{~g} / \mathrm{kg} \\
\text { phosphorus) induced calcification }\end{array} \\
\frac{\text { In vitro }}{\text { MOVAS cell line }} \\
-\quad \mathrm{CaCl}_{2}(1.8 \mathrm{mM})+\beta-\mathrm{GP} \\
\quad(1.25-2.5 \mathrm{mM}) \text { induced } \mathrm{VCN}\end{array}$ & $\begin{array}{l}\text { HPD/ } \beta \text {-GP: } \uparrow(T r p 53 i n, \text { Igfbp3, Hmox1, Adrb2, } \\
\text { Atg16l1, LC3-II), } \uparrow \text { Vascular calcification } \\
\text { Rapamycin: } \uparrow \uparrow L C 3-I I, \downarrow \text { VSMC calcification, } \\
\downarrow \text { Runx2 } \\
\text { 3-MA: } \uparrow \uparrow V S M C \text { calcification }\end{array}$ & $\begin{array}{l}\text { Uremic media calcification increases } \\
\text { autophagy, which acts as a protective } \\
\text { mechanism. Enhancing autophagy with } \\
\text { rapamycin, attenuates VCN }\end{array}$ \\
\hline $\begin{array}{c}{[54]} \\
\text { (Zhan et al., 2014) }\end{array}$ & $\begin{array}{c}\text { Genetic and pharmacological: } \\
\text { mTOR }\end{array}$ & $\begin{array}{l}\frac{\text { In vitro }}{\text { Primary mouse aortic VSMCs }} \\
-\quad \mathrm{CaCl}_{2}(1.8 \mathrm{mM})+\beta-\mathrm{GP}(10 \mathrm{mM}) \\
\quad \text { induced calcification }\end{array}$ & $\begin{array}{l}\beta \text {-GP: } \uparrow \text { mTOR } \\
\text { mTOR-siRNA: } \downarrow \text { (ALP, OC), } \downarrow \text { Calcification } \\
\text { Rapamycin: } \downarrow \text { (mTOR, p70S6k, p-mTOR } \\
\text { p-p70s2448, } \\
\text { Adiponectin: } \downarrow \text { mTOR, } \downarrow \text { ALP, } \downarrow \text { Calcification }\end{array}$ & $\begin{array}{l}\text { mTOR is involved in the signal } \\
\text { transduction of VCN, whilst inhibiting its } \\
\text { activity attenuates VSMC mineralization } \\
\text { and osteoblastic differentiation }\end{array}$ \\
\hline
\end{tabular}


Table 1. Cont.

\begin{tabular}{|c|c|c|c|c|}
\hline Authors & $\begin{array}{c}\text { Type of } \\
\text { Intervention/Modulation }\end{array}$ & Methods/Matrix & Molecular Outcome & Study Conclusion \\
\hline $\begin{array}{c}{[56]} \\
\text { (Zhao et al., 2015b) }\end{array}$ & $\begin{array}{l}\text { Pharmacological: } \\
\text { Rapamycin } \\
\text { Genetic: } \\
\text { Klotho }\end{array}$ & $\begin{array}{l}\frac{\text { In vivo }}{\text { Wistar rats }} \\
-\quad \text { Adenine }(0.75 \%) \text {-induced CRF } \\
\text { Klotho knockout mice (C57BL/6- }-\mathrm{C} 3 \mathrm{H} / \mathrm{J} \\
\text { background }) \\
\text { In vitro } \\
\text { Aortic rings }\left(\mathrm{Kl}^{-/-} \text {and WT mice }\right) \\
\mathrm{T} / \mathrm{G} \mathrm{HASMCs} \text { and BASMCs } \\
-\quad \mathrm{CaCl}_{2}(1.4 \mathrm{mM})+\mathrm{Pi} \\
\quad(3 \mathrm{mM}) \text { induced calcification }\end{array}$ & $\begin{array}{l}\text { High Pi: } \uparrow(\mathrm{p}-\mathrm{mTOR}, \mathrm{p}-\mathrm{S} 6 \mathrm{~K}), \downarrow \text { Klotho } \\
\text { mTOR overexpression: } \uparrow \text { Calcification, } \downarrow \text { Klotho } \\
\text { Kinase-dead mTOR: } \downarrow \text { Calcification } \\
\text { Rapamycin: } \downarrow \text { Calcification, } \downarrow(\text { Msx2, Cbfo- } 1 \text {, } \\
\text { aggrecan, Sox } 9), \uparrow(\mathrm{M} g p, \text { Oph), } \uparrow \text { Klotho } \\
\text { 3-MA: partially abrogates effects of rapamycin } \\
\text { Klotho overexpression: } \downarrow \text { Calcification } \\
\text { Klotho-siRNA: Abrogates effects of rapamycin }\end{array}$ & $\begin{array}{l}\text { Rapamycin attenuates VCN by } \\
\text { upregulating Klotho via mTOR inhibition }\end{array}$ \\
\hline $\begin{array}{c}{[57]} \\
\text { (He et al., 2019b) }\end{array}$ & $\begin{array}{l}\text { Pharmacological: } \\
\text { Bavachin }\end{array}$ & $\begin{array}{l}\frac{\text { In vitro }}{\text { Primary HASMCs }} \\
-\quad \beta \text {-GP }(10 \mathrm{mM}) \text { induced calcification }\end{array}$ & $\begin{array}{l}\text { Bavachin: } \uparrow(\text { LC3-II, Beclin1), } \downarrow \text { p-mTOR, } \downarrow \text { (Runx2, } \\
\text { BMP2, OPN, OPG), } \downarrow \text { (Wnt3A, } \beta \text {-catenin) } \\
\text { Wortmannin: Abrogates the effect of bavachin } \\
\text { Atg7 siRNA (in vitro): Abrogates the effect } \\
\text { of bavachin }\end{array}$ & $\begin{array}{l}\text { Bavachin suppresses HASMC } \\
\text { calcification by acting on } \\
\text { Atg7/mTOR-mediated } \\
\text { autophagy signalling }\end{array}$ \\
\hline $\begin{array}{c}{[58]} \\
\text { (Chen et al., 2020a) }\end{array}$ & $\begin{array}{l}\text { Pharmacological: } \\
\text { Melatonin }\end{array}$ & $\begin{array}{l}\frac{\text { In vitro }}{\text { Primary rat VSMCs }} \\
-\quad \mathrm{CaCl}_{2}(1.8 \mathrm{mM})+\beta-\mathrm{GP}(10 \mathrm{mM}) \\
\quad \text { induced VCN }\end{array}$ & $\begin{array}{l}\text { Melatonin: } \downarrow(\text { VCN, Runx2, ALP activity); } \uparrow(\text { LC3-II, } \\
\text { Beclin1, p-AMPK, p-ULK1), } \downarrow \text { p-mTOR } \\
\text { Compound C \& MHY1485: Abrogated the effect of } \\
\text { melatonin, } \uparrow \text { VCN }\end{array}$ & $\begin{array}{l}\text { Melatonin attenuates VSMC calcification } \\
\text { in vitro by acting on the } \\
\text { AMPK/mTOR pathway }\end{array}$ \\
\hline $\begin{array}{c}{[59]} \\
\text { (Ma et al., 2019b) }\end{array}$ & $\begin{array}{l}\text { Pharmacological: } \\
\text { Metformin }\end{array}$ & $\begin{array}{l}\frac{\text { In vitro }}{\text { Primary rat aortic VSMCs }} \\
-\quad \mathrm{CaCl}_{2}(1.8 \mathrm{mM})+\beta-\mathrm{GP}(10 \mathrm{mM}) \\
\quad \text { induced calcification }\end{array}$ & $\begin{array}{l}\text { Metformin: } \downarrow \text { VSMC calcification, } \downarrow \text { (Runx2, BMP2), } \\
\uparrow p-A M P K, \downarrow \text { PDK4, } \uparrow \text { LC3-II/LC3-I, } \downarrow \text { p62, } \uparrow(T F A M, \\
\text { NRF1, PGC- }-1 \text { a) } \\
\text { Compound C: Abrogates protective effects } \\
\text { of metformin } \\
\text { Atg5 siRNA (in vitro): Abrogates protective effects } \\
\text { of metformin }\end{array}$ & $\begin{array}{l}\text { Metformin-mediated AMPK activation } \\
\text { attenuates Pi-induced calcification and } \\
\text { restores disrupted mitochondrial } \\
\text { biogenesisMitophagy regulates } \\
\text { metformin-induced } \\
\text { mitochondrial biogenesis }\end{array}$ \\
\hline $\begin{array}{c}{[60]} \\
\text { (Chen et al., 2020b) }\end{array}$ & $\begin{array}{l}\text { Pharmacological: } \\
\text { Intermedin } 1-53\end{array}$ & 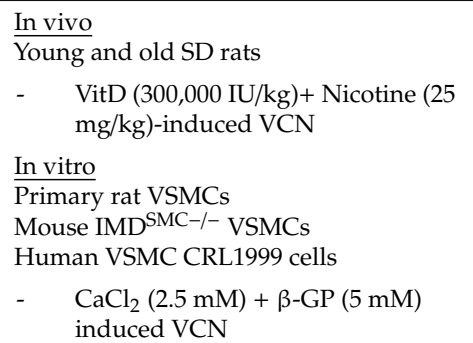 & 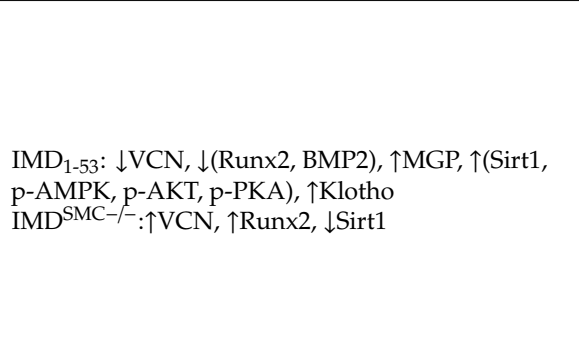 & $\begin{array}{l}\mathrm{IMD}_{1-53} \text { plays a protective role in } \mathrm{VCN} \\
\text { by upregulating Sirt1 }\end{array}$ \\
\hline
\end{tabular}


Table 1. Cont.

\begin{tabular}{|c|c|c|c|c|}
\hline Authors & $\begin{array}{c}\text { Type of } \\
\text { Intervention/Modulation }\end{array}$ & Methods/Matrix & Molecular Outcome & Study Conclusion \\
\hline $\begin{array}{c}{[61]} \\
\text { (Xu et al., 2017) }\end{array}$ & $\begin{array}{l}\text { Pharmacological: } \\
\text { Ghrelin }\end{array}$ & 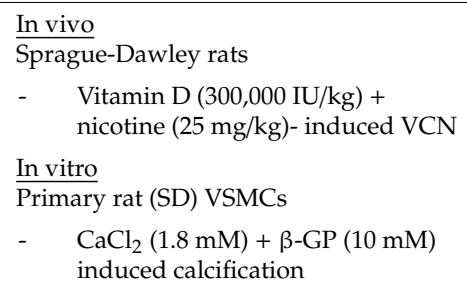 & $\begin{array}{l}\text { Ghrelin: } \downarrow \text { VCN, } \downarrow \text { ALP activity, } \uparrow(\text { LC3-II, Beclin1), } \\
\uparrow p \text {-AMPK } \\
\text { 3-MA: Abrogates protective effect of Ghrelin } \\
\text { Compound C: Abrogates the effect of Ghrelin on } \\
\text { VCN and autophagy }\end{array}$ & $\begin{array}{l}\text { Ghrelin attenuates VCN by inducing } \\
\text { autophagy through AMPK activation }\end{array}$ \\
\hline $\begin{array}{c}{[62]} \\
\text { (Wei et al., 2020) }\end{array}$ & $\begin{array}{c}\text { Pharmacological: } \\
\text { Mitochondrial-derived peptide } \\
\text { MOTS-c }\end{array}$ & $\begin{array}{l}\text { In vivo } \\
\text { Sprague-Dawley rats } \\
-\quad \text { Vitamin } \mathrm{D}(300,000 \mathrm{IU} / \mathrm{kg})+ \\
\quad \text { nicotine }(5 \mathrm{~mL} / \mathrm{kg}) \text { induced VCN }\end{array}$ & $\begin{array}{l}\text { MOTS-c: } \downarrow V C N, \downarrow \text { (Aortic calcium content, } \\
\text { ALP activity), } \uparrow \text { p-AMPK, } \downarrow \text { (AT-1 receptor, } \\
\text { ET-B receptor) }\end{array}$ & $\begin{array}{l}\text { MOTS-c attenuates VCN by activating } \\
\text { the AMPK pathway }\end{array}$ \\
\hline $\begin{array}{l}{[63]} \\
\text { (Ciceri et al., 2015) }\end{array}$ & $\begin{array}{c}\text { Non-pharmacological: } \\
\text { intermittent suspension (IS) } \\
\text { of Pi } \\
\text { Pharmacological: } \\
\text { Valproic acid (IS) }\end{array}$ & $\begin{array}{l}\text { In vitro } \\
\text { Primary rat } \mathrm{VSMCs} \\
-\quad \mathrm{CaCl}_{2}(1.8 \mathrm{mM})+\mathrm{Pi} \\
\quad(5 \mathrm{mM}) \text {-induced calcification }\end{array}$ & $\begin{array}{l}\text { IS Pi: } \downarrow \text { VCN, } \downarrow \text { (Runx } 2), \uparrow(\text { LC3-II), } \\
\uparrow \text { Autophagic flux } \\
\text { IS valproic acid: = VCN }\end{array}$ & $\begin{array}{l}\text { Intermittent Pi increases autophagic flux } \\
\text { and ameliorates VSMC calcification, } \\
\text { intermittent valproic acid treatment, } \\
\text { however, does not attenuate VCN }\end{array}$ \\
\hline $\begin{array}{c}{[65]} \\
\text { (Yao et al., 2017b) }\end{array}$ & $\begin{array}{l}\text { Genetic: } \\
\text { Nrf2 }\end{array}$ & $\begin{array}{l}\text { In vitro } \\
\text { Primary rat }(\mathrm{SD}) \text { aortic VSMCs } \\
-\quad \mathrm{CaCl}_{2}(1.8 \mathrm{mM})+\mathrm{High} \mathrm{Pi} \\
\quad(1.4-2.5 \mathrm{mM}) \text {-induced calcification }\end{array}$ & $\begin{array}{l}\text { Nrf2-siRNA: } \uparrow \text { Calcification, } \uparrow(\text { BMP2, Runx2), } \\
\downarrow \text { Autophagosomes, } \downarrow \text { LC3-II/LC3-I } \\
\text { Nrf2 overexpression: } \downarrow \text { Calcification, } \downarrow \text { (BMP2, } \\
\text { Runx2), } \uparrow \text { Autophagosomes, } \uparrow \text { LC3-II/LC3-I }\end{array}$ & $\begin{array}{l}\text { Activation of the Nrf2-ARE pathway } \\
\text { alleviates hyperphosphatemia-induced } \\
\text { calcification, possibly by } \\
\text { inducing autophagy }\end{array}$ \\
\hline $\begin{array}{c}{[66]} \\
\text { (Peng et al., 2017b) }\end{array}$ & $\begin{array}{l}\text { Pharmacological: } \\
\text { Oestrogen }\end{array}$ & 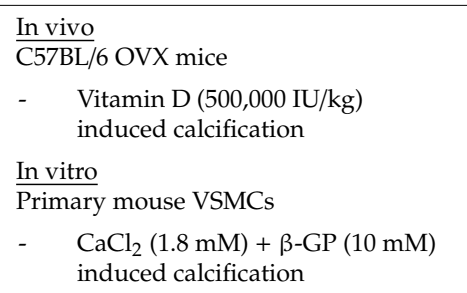 & $\begin{array}{l}\text { Oestrogen: } \downarrow \text { Calcification, } \downarrow \text { (Runx2, ALP activity), } \\
\uparrow \text { (Atg5, LC3-I, LC3-II) } \\
\text { 3-MA: Counteracted effects of oestrogen } \\
\text { ER }_{\alpha} \text {-antagonist/knockdown: } \uparrow(\text { Runx2, ALP } \\
\text { activity), } \downarrow \text { (LC3-I, LC3-II) }\end{array}$ & $\begin{array}{l}\text { Oestrogen-induced autophagy inhibits } \\
\text { arterial calcification through the } \\
\qquad \mathrm{ER}_{\alpha} \text { pathway }\end{array}$ \\
\hline
\end{tabular}


Table 1. Cont.

\begin{tabular}{|c|c|c|c|c|}
\hline Authors & $\begin{array}{c}\text { Type of } \\
\text { Intervention/Modulation }\end{array}$ & Methods/Matrix & Molecular Outcome & Study Conclusion \\
\hline $\begin{array}{c}{[67]} \\
\text { (Liu et al., 2014) }\end{array}$ & $\begin{array}{l}\text { Pharmacological: } \\
\text { Atorvastatin }\end{array}$ & $\begin{array}{l}\frac{\text { In vitro }}{\text { Primary rat (SD) VSMCs }} \\
-\quad \begin{aligned} \mathrm{CaCl}_{2}(1.8 \mathrm{mM})+\mathrm{TGF}-\beta 1(2 \\
\mathrm{ng} / \mathrm{mL}) \text {-induced calcification }\end{aligned}\end{array}$ & $\begin{array}{l}\text { Atorvastatin: } \downarrow \text { Calcification; } \downarrow \text { (ALP, BMP2, } \\
\text { Osteocalcin), } \downarrow \text { Nuclear } \beta \text {-catenin expression, } \\
\uparrow \text { Autophagy; } \uparrow \text { (Beclin1, Atg5, LC3-II/LC3-I ratio) } \\
\text { Autophagy inhibitors (3-MA, Chloroquine, } \mathrm{NH}_{4} \mathrm{Cl} \text {, } \\
\text { bafilomycin A1): Suppresses effect of atorvastatin } \\
\text { on autophagy and calcification } \\
\beta \text {-catenin overexpression: Abrogates effect of } \\
\text { atorvastatin } \\
\beta \text {-catenin inhibitor JW74: } \\
\uparrow \text { Effect of atorvastatin }\end{array}$ & $\begin{array}{l}\text { Atorvastatin suppresses TGF- } \beta 1 \text {-induced } \\
\text { VCN by inducing autophagy via } \\
\text { downregulation of the } \beta \text {-catenin pathway }\end{array}$ \\
\hline $\begin{array}{c}{[68]} \\
\text { (Xu et al., 2019b) }\end{array}$ & $\begin{array}{l}\text { Pharmacological: } \\
\text { miRNA-30b }\end{array}$ & $\begin{array}{l}\frac{\text { In vivo }}{\text { Sprague-Dawley rats }} \\
-\quad(5 / 6 \text { nephrectomy }(\mathrm{Nx})+\text { high Pi } \\
\quad(1.2 \%) \text { diet)-induced CKD } \\
\text { In vitro } \\
\text { VSMCs } \\
-\quad\left(\mathrm{CaCl}_{2}(1.8 \mathrm{mM})+\beta-\mathrm{GP}(10 \mathrm{mM})\right. \\
\quad \text { induced calcification }\end{array}$ & $\begin{array}{l}\text { miR-30b mimic: } \downarrow \text { VCN }, \downarrow(\text { SOX9, Msx2, Runx2) } \\
\uparrow(\text { LC3-II/LC3-I, Beclin1), } \uparrow \text { Mitochondrial } \\
\text { membrane potential (MMP) } \\
\text { miR-30b inhibitor + Rapamycin: no } \\
\text { mTOR inhibition }\end{array}$ & $\begin{array}{l}\text { miRNA-30b protects against } \mathrm{VCN} \text { by } \\
\text { promoting MMP and autophagy, via } \\
\text { targeted inhibition of SOX9 or negatively } \\
\text { regulating the mTOR pathway }\end{array}$ \\
\hline $\begin{array}{c}{[69]} \\
\text { (Zhang et al., 2020b) }\end{array}$ & $\begin{array}{c}\text { Genetic: } \\
\text { Anti-differentiation non-coding } \\
\text { RNA (ANCR) }\end{array}$ & 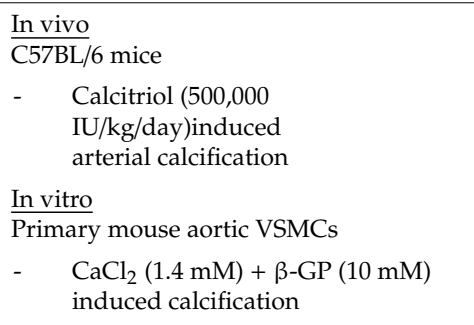 & $\begin{array}{l}\text { ANCR overexpression: } \downarrow \text { Calcification, } \downarrow \text { (BMP2, } \\
\text { Runx2), } \uparrow(\text { Atg5, LC3-II, LC3-I) }\end{array}$ & $\begin{array}{c}\text { ANCR attenuates VCN and VSMC } \\
\text { osteochondrogenic differentiation by } \\
\text { activating autophagy }\end{array}$ \\
\hline $\begin{array}{c}{[70]} \\
\text { (Shi et al., 2020) }\end{array}$ & $\begin{array}{c}\text { Genetic: } \\
\text { Klotho knockout } \\
\text { Beclin1 overexpression }\end{array}$ & $\begin{array}{l}\text { In vivo } \\
\text { Becn1F121A knock-in mice (129 S1/SVlmJ) } \\
(B K) \\
\alpha K l o t h o \text { homozygous knockout mice (129 } \\
\text { S1/SVlmJ) }(k l / k l) \\
\text { BK/BK; } k l / k l \text { mice } \\
-\quad \text { Age-induced calcification } \\
\quad(10 \text { weeks })\end{array}$ & $\begin{array}{l}\alpha \text { Klotho homozygous knockout }(\mathrm{kl} / \mathrm{kl}) \text { : } \\
\uparrow \text { Calcification } \\
\text { Beclin1 homozygous overexpression: Attenuates } \\
\text { calcification in } \alpha \text { Klotho knockout mice }\end{array}$ & $\begin{array}{l}\text { Beclin1 overexpression alleviates } \\
k l / k l \text {-induced vascular calcification }\end{array}$ \\
\hline
\end{tabular}


Table 1. Cont.

\begin{tabular}{|c|c|c|c|c|}
\hline Authors & $\begin{array}{c}\text { Type of } \\
\text { Intervention/Modulation }\end{array}$ & Methods/Matrix & Molecular Outcome & Study Conclusion \\
\hline $\begin{array}{c}{[71]} \\
\text { (Ciceri et al., 2019) }\end{array}$ & $\begin{array}{l}\text { Non-pharmacological: } \\
\text { Iron citrate }\end{array}$ & $\begin{array}{l}\text { In vitro } \\
\text { Primary rat aortic VSMCs } \\
-\quad \mathrm{CaCl}_{2}(1.8 \mathrm{mM})+\mathrm{Pi} \\
\quad(5 \mathrm{mM}) \text {-induced calcification }\end{array}$ & $\begin{array}{l}\text { Iron citrate: } \downarrow \text { Calcification, } \uparrow \text { Autophagosomes, } \\
\uparrow \text { Autophagic flux, } \uparrow \text { LC3-Ii } \beta\end{array}$ & $\begin{array}{l}\text { Iron citrate blocks the progression of } \\
\text { calcification by inducing autophagy }\end{array}$ \\
\hline $\begin{array}{c}{[72]} \\
\text { (Yang et al., 2019) }\end{array}$ & $\begin{array}{l}\text { Pharmacological: } \\
\text { Advanced glycation } \\
\text { end-products (AGEs) }\end{array}$ & $\begin{array}{l}\frac{\text { In vitro }}{\text { Primary VSMCs }} \\
-\quad \mathrm{CaCl}_{2}(1.8 \mathrm{mM})+\beta-\mathrm{GP}(10 \mathrm{mM}) \\
\quad \text { induced calcification }\end{array}$ & $\begin{array}{l}\text { AGEs: } \uparrow \text { HIF- } 1 \alpha, \uparrow \text { PDK4, } \uparrow \text { LC3-II, } \downarrow \text { p62, } \\
\uparrow(\text { Autophagosomes, autolysosomes), } \uparrow \text { LC3-II \& } \\
\text { LAMP1 colocalization } \\
\text { Dichloroacetic acid (PDK inhibitor) + AGEs: } \\
\downarrow \text { LC3-II, } \uparrow \text { p } 62 \\
\text { Attenuates effect of AGEs } \\
\text { Rapamycin: } \downarrow \text { Calcification, } \downarrow \text { Runx2 } \\
\text { 3-MA: } \uparrow \uparrow C \text { Calcification }\end{array}$ & $\begin{array}{l}\text { AGEs induce autophagy through } \\
\text { HIF-1 } \alpha / \text { PDK4 signalling, which has } \\
\text { protective effects against } \\
\text { AGEs-induced VCN }\end{array}$ \\
\hline $\begin{array}{c}{[73]} \\
\text { (Liu et al., 2020b) }\end{array}$ & $\begin{array}{l}\text { Pharmacological: } \\
\text { Advanced glycation } \\
\text { end-products (AGEs) }\end{array}$ & $\begin{array}{l}\frac{\text { In vitro }}{\text { A7R5 cells }} \\
\text { Wild-type rat aortic segments }\end{array}$ & $\begin{array}{l}\text { AGEs: } \uparrow \text { VCN, } \uparrow(\text { BMP2, RUNX2), } \downarrow \text { (BECN, LC3-II, } \\
\text { p-AMPK } \downarrow), \uparrow p-m T O R\end{array}$ & $\begin{array}{l}\text { AGEs induce VSMC calcification by } \\
\text { suppressing autophagy through action } \\
\text { on the AMPK/mTOR signalling pathway }\end{array}$ \\
\hline $\begin{array}{c}{[74]} \\
\text { (Chen et al., 2018) }\end{array}$ & $\begin{array}{l}\text { Pharmacological: } \\
\text { Agonist-CD137, SP600125 }\end{array}$ & $\begin{array}{l}\frac{\text { In vivo }}{\text { ApoE }^{-/}} \text {mice } \\
-\quad \text { Western diet induced VCN } \\
\text { In vitro } \\
\text { Primary VSMCs from C57BL/6J mice } \\
-\quad \mathrm{CaCl}_{2}(1.8 \mathrm{mM})+\beta-\mathrm{GP}(10 \mathrm{mM}) \\
\quad \text { induced } \mathrm{VCN}\end{array}$ & $\begin{array}{l}\text { Agonist-CD137: } \uparrow \text { AMC and osteogenic VSMC } \\
\text { phenotype transition; } \uparrow(\text { Beclin1, p62, LC3B), } \\
\text { autophagosome accumulation } \\
\text { SP600125 (JNK-inhibition): Attenuated the effect } \\
\text { of agonist-CD137 }\end{array}$ & $\begin{array}{l}\text { CD137 activation disrupts autophagic } \\
\text { flux and accelerates calcification through } \\
\text { the action of the JNK phosphorylation }\end{array}$ \\
\hline $\begin{array}{c}{[75]} \\
(\text { Xu et al., 2020) }\end{array}$ & $\begin{array}{c}\text { Genetic: } \\
\text { O-GlcNAc transferase (OGT) }\end{array}$ & $\begin{array}{l}\text { In vivo } \\
\text { Sprague-Dawley rats } \\
-\quad(5 / 6 \text { nephrectomy }(\mathrm{Nx})+\text { high } \mathrm{Pi} \\
\quad(1.2 \%) \text { diet)-induced CKD } \\
\text { In vitro } \\
\text { Rat } \mathrm{VSMCs} \\
-\quad \mathrm{CaCl}_{2}(1.8 \mathrm{mM})+\beta-\mathrm{GP}(10 \mathrm{mM}) \\
\quad \text { induced calcification }\end{array}$ & $\begin{array}{l}\text { shOGT: } \downarrow \text { VCN } \\
\text { OGT overexpression: } \uparrow(\text { YAP glycosylation, YAP } \\
\text { protein expression \& nuclear translocation), } \downarrow \text { YAP } \\
\text { phosphorylation, } \downarrow \text { (Autophagosomes, } \\
\text { LC3-II/LC3-I), } \uparrow \text { p2, } \uparrow \text { Calcification, } \uparrow \text { Runx2, } \\
\downarrow \alpha-S M A \\
\text { Rapamycin or YAP silencing: ameliorates the } \\
\text { effects of OGT overexpression } \\
\text { YAP overexpression: } \downarrow \text { (Autophagosomes, } \\
\text { LC3-II/LC3-I), } \uparrow p 62, \uparrow C \text { Calcification, } \uparrow \text { Runx2, } \\
\downarrow \alpha-S M A\end{array}$ & $\begin{array}{l}\text { Upregulated OGT in high Pi diet-fed } \\
\text { CKD rats, promotes glycosylation of YAP } \\
\text { to inhibit autophagy, facilitating VCN }\end{array}$ \\
\hline
\end{tabular}


Table 1. Cont.

\begin{tabular}{|c|c|c|c|c|}
\hline Authors & $\begin{array}{c}\text { Type of } \\
\text { Intervention/Modulation }\end{array}$ & Methods/Matrix & Molecular Outcome & Study Conclusion \\
\hline $\begin{array}{c}{[76]} \\
\text { (Shiozaki et al., 2020) }\end{array}$ & $\begin{array}{l}\text { Non-pharmacological: } \\
\text { (Un)saturated fatty acids } \\
\text { (SFA/UFA) }\end{array}$ & $\begin{array}{l}\text { In vivo } \\
\text { SMMHC-CreER(T2); } \\
\text { Atg5(lox/lox) mice SMC } \\
\text { Scd1/2 KO; Gpat4 triple KO mice } \\
\text { SMC-Scd1/2 KO } \\
\text { In vitro } \\
\text { Humanan and mouse VSMCs, MOVAS-1 } \\
\text { cell line } \\
\text { GFP-LC3-RFP-LC3 } \\
\text { G probe } \\
\text { Gpat4 siRNA } \\
-\quad \mathrm{CaCl}_{2}(1.8 \mathrm{mM})+\mathrm{Pi}(2.6 \mathrm{mM}) \\
\quad \text { induced calcification }\end{array}$ & $\begin{array}{l}\text { Atg5 KO (in vivo/in vitro): } \uparrow \text { VCN, } \uparrow p 62, \downarrow \text { LC3-II } \\
\text { SFAs: } \downarrow \text { Autophagic flux ( } \uparrow \mathrm{GFP} / \mathrm{RFP}), \\
\text { Accumulation of isolation membranes } \\
\text { Gpat4 KO: Attenuates autophagy inhibition by } \\
\text { SFAs } \\
\text { Unsaturated LPAs: } \downarrow \text { SFA-induced LC3-II } \\
\text { accumulation, } \downarrow \text { Calcification } \\
\text { SMC-Scd1/2 KO: } \uparrow \text { Saturated LPAs, } \uparrow \uparrow C a l c i f i c a t i o n, \\
\uparrow(p 62, \text { Fam134b2) } \\
\text { SMC-Scd1/2 KO; Gpat4 triple KO: Blocks SCD } \\
\text { deficiency-induced VCN and autophagic flux } \\
\text { inhibition }\end{array}$ & $\begin{array}{l}\text { Excess saturated LPAs cause omegasome } \\
\text { formation, which in turn produces and } \\
\text { accumulates isolation membranes, blocks } \\
\text { autophagic flux and causes VCNGpat4 } \\
\text { converts SFAs to saturated LPAs, which } \\
\text { makes Gpat4 an interesting target in VCN }\end{array}$ \\
\hline $\begin{array}{c}\text { [77] } \\
\text { (Zhu et al., 2020b) }\end{array}$ & $\begin{array}{l}\text { Non-pharmacological: } \\
\text { Lactate }\end{array}$ & $\begin{array}{l}\text { In vivo } \\
\text { Wistar rats } \\
\text { Vitamin } \mathrm{D}(300,000 \mathrm{IU} / \mathrm{kg})+\text { nicotin } \\
(25 \mathrm{mg} / \mathrm{kg}) \text { induced VCN } \\
\text { In vitro } \\
\text { Rat aortic rings } \\
\text { Primary rat }(\mathrm{SD}) \text { aortic VSMCs } \\
-\quad \mathrm{CaCl}_{2}(1.4 \mathrm{mM})+\beta-\mathrm{GP}(10 \mathrm{mM}) \\
\quad \text { induced calcification }\end{array}$ & $\begin{array}{l}\text { Lactate: } \uparrow \text { Calcification; } \uparrow(B M P 2, \text { Runx2), } \downarrow \alpha-S M A, \\
\downarrow \text { Autophagy/Mitophagy; } \downarrow \text { (LC3-II, BNIP3), } \uparrow \text { p } 62, \\
\uparrow \text { Mitochondrial fission; } \uparrow \text { mito-Drp1, } \downarrow \text { OPA1, } \\
\uparrow N R 4 A 1 \\
\text { NR4A1 silencing/knockdown: Abrogates effects of } \\
\text { Lactate, } \uparrow \text { Mitochondrial clearance/Mitophagy; } \\
\uparrow B N I P 3, \downarrow(\text { TOMM20, TOMM40), } \uparrow \text { Mitochondrial } \\
\text { respiration, } \downarrow \text { mitochondrial swelling, } \\
\uparrow \text { Autophagosome-mitochondria fusion }\end{array}$ & $\begin{array}{l}\text { Lactate inhibits BNIP3-mediated } \\
\text { mitophagy via the } \\
\text { NR4A1/DNA-PKcs/p53 pathway, } \\
\text { enhancing mitochondrial fission and } \\
\text { therefore accelerating VCN }\end{array}$ \\
\hline $\begin{array}{c}{[78]} \\
\text { (Zhu et al., 2019b) }\end{array}$ & $\begin{array}{l}\text { Non-pharmacological: } \\
\text { Lactate }\end{array}$ & $\begin{array}{l}\frac{\text { In vitro }}{\text { Primary rat }(\mathrm{SD}) \text { aortic VSMCs }} \\
-\quad \mathrm{CaCl}_{2}(1.4 \mathrm{mM})+\beta-\mathrm{GP}(10 \mathrm{mM}) \\
\quad \text { induced calcification }\end{array}$ & $\begin{array}{l}\text { Lactate: } \uparrow \text { Calcification; } \uparrow(B M P 2, \text { Runx2, ALP } \\
\text { activity), } \downarrow \text { Mitochondrial function \& biogenesis, } \\
\downarrow \text { Autophagic flux; } \downarrow \text { Autolysosomes, } \downarrow \text { LC3-II, } \uparrow 62, \\
\downarrow \text { Mitochondrial clearance/Mitophagy; } \uparrow \text { TOMM20, } \\
\downarrow \text { BNIP3 } \\
\text { BNIP3 overexpression: } \downarrow \text { Calcification; } \\
\downarrow \text { (BMP2, Runx2) }\end{array}$ & $\begin{array}{c}\text { Lactate accelerates VCN by facilitating } \\
\text { mitochondrial dysfunction and inhibiting } \\
\text { BNIP3-mediated mitophagy }\end{array}$ \\
\hline \multicolumn{5}{|c|}{ Modulation of lysosomal function in vascular calcification } \\
\hline $\begin{array}{c}{[55]} \\
\text { (Liu et al., 2020a) }\end{array}$ & $\begin{array}{l}\text { Non-pharmacological: } \\
\text { Hydroxyapatite (HAP) }\end{array}$ & $\begin{array}{l}\frac{\text { In vitro }}{\text { A7R5 cells }} \\
-\quad \text { Pi/HAP-induced calcification }\end{array}$ & $\begin{array}{l}\text { Pi + HAP: } \uparrow \text { Calcification, } \uparrow(\text { Runx2, BMP2), } \\
\downarrow \text { Lysosome integrity }\end{array}$ & $\begin{array}{c}\text { Adhesion of HAP causes cell injury, } \\
\text { which leads cell damage and decreased } \\
\text { lysosomal integrity, therefore } \\
\text { contributing to the development of VCN }\end{array}$ \\
\hline
\end{tabular}


Table 1. Cont.

\begin{tabular}{|c|c|c|c|c|}
\hline Authors & $\begin{array}{c}\text { Type of } \\
\text { Intervention/Modulation }\end{array}$ & Methods/Matrix & Molecular Outcome & Study Conclusion \\
\hline $\begin{array}{c}{[79]} \\
\text { (Bhat et al., 2020c) }\end{array}$ & $\begin{array}{c}\text { Genetic: } \\
\text { Mcoln1 }{ }^{-/-} \\
\text {Pharmacological: } \\
\text { MLSA-1, Verapamil }\end{array}$ & 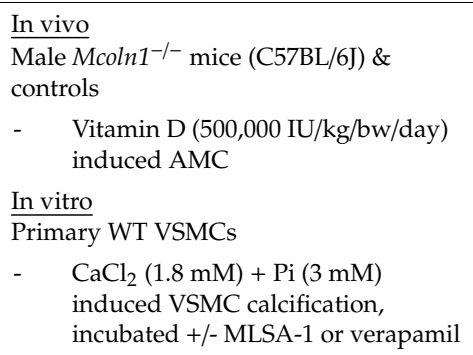 & $\begin{array}{l}\text { Mcoln1 } 1^{-/-}: \uparrow A M C \text { and SMC osteogenic phenotype } \\
\text { transition (in vivo), } \downarrow \text { Lysosomal trafficking/MVB } \\
\text { colocalization, } \uparrow \text { sEV secretion } \\
\text { Effect on lysosomal trafficking: } \\
\text { Verapamil: } \downarrow \\
\text { MLSA-1: } \uparrow\end{array}$ & $\begin{array}{l}\text { Mcoln1/TRPML1 deletion impairs normal } \\
\text { lysosomal trafficking, leading to } \\
\text { enhanced sEV secretion, contributing to } \\
\text { the development of AMC }\end{array}$ \\
\hline $\begin{array}{c}{[80]} \\
\text { (Bhat et al., 2020a) }\end{array}$ & $\begin{array}{l}\text { Genetic: } \\
\text { Asah1 } 1^{--}\end{array}$ & 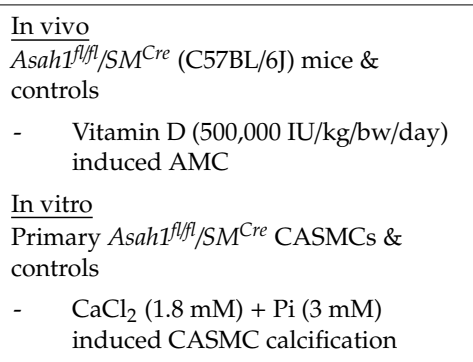 & 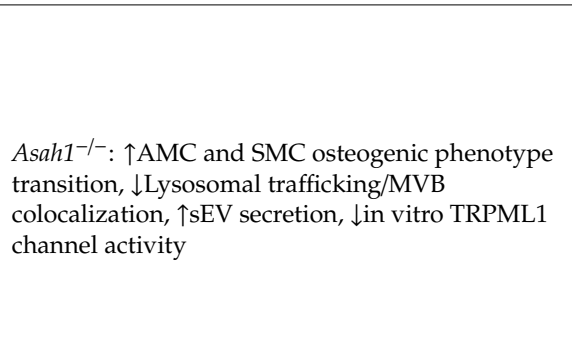 & $\begin{array}{l}\text { Asah1 gene deletion impairs TRPML1 } \\
\text { channel activation which causes } \\
\text { impaired lysosomal trafficking, thereby } \\
\text { enhancing AMC }\end{array}$ \\
\hline $\begin{array}{c}{[81]} \\
\text { (Bhat et al., 2020b) }\end{array}$ & $\begin{array}{c}\text { Genetic: } \\
\text { sphingomyelin } \\
\text { phosphodiesterase } 1 \text { (Smpd1) } \\
\text { overexpression }\end{array}$ & $\begin{array}{l}\text { In vivo } \\
\text { Smpd1 }{ }^{\text {trg }} / \mathrm{SM}^{\text {cre }} \text { mice }(\mathrm{C} 57 \mathrm{BL} / 6 \mathrm{~J}) \\
-\quad \text { Vitamin } \mathrm{D}(500,000 \mathrm{IU} / \mathrm{kg} / \mathrm{bw} / \text { day }) \\
\quad \text { induced AMC } \\
\text { In vitro } \\
\text { Primary mouse CASMCs } \\
-\quad \mathrm{CaCl}_{2}(1.8 \mathrm{mM})+\mathrm{Pi}(3 \mathrm{mM}) \\
\quad \text { induced calcification }\end{array}$ & $\begin{array}{l}\text { Smpd1 } \operatorname{trg} / S M^{c r e} \text { mice: } \uparrow \text { Calcification; } \uparrow(\mathrm{OSP}, \\
\text { Runx2), } \downarrow \text { SM22- } \alpha, \downarrow \text { Lysosomal trafficking/MVB } \\
\text { colocalization, } \uparrow \text { sEV secretion, } \uparrow \text { Arterial stiffness; } \\
\text { disorganized elastic lamellae } \\
\text { Amitriptyline: Abrogates effects of } \\
\text { Smpd1 overexpression }\end{array}$ & $\begin{array}{l}\text { Lysosomal overexpression of } S m p d 1 \\
\text { enhances the secretion of sEVs and } \\
\text { facilitates osteogenic phenotype switch of } \\
\text { SMCs, initiating arterial } \\
\text { medial calcification }\end{array}$ \\
\hline $\begin{array}{c}\text { [82] } \\
\text { (Sudo et al., 2015) }\end{array}$ & $\begin{array}{l}\text { Pharmacological: } \\
\text { 7-ketocholesterol (7-KC) }\end{array}$ & $\begin{array}{l}\frac{\text { In vitro }}{\text { Human aortic } \mathrm{SMCs}} \\
-\quad \mathrm{CaCl}_{2}(1.8 \mathrm{mM})+\mathrm{Pi}(3 \mathrm{mM}) \\
\quad \text { induced calcification }\end{array}$ & $\begin{array}{l}\text { 7-KC: } \uparrow \text { Calcium deposition/calcification, } \\
\text { Autophagosome accumulation, } \uparrow(\mathrm{p} 62, \mathrm{LC} 3-\mathrm{II}), \\
\downarrow \text { Mature cathepsin B and D } \\
\text { Atg5 siRNA: = Calcium deposition ( vs. 7-KC) } \\
\text { Lysosomal protease inhibitors: } \uparrow \uparrow \text { Calcium } \\
\text { deposition (vs. 7-KC) }\end{array}$ & $\begin{array}{c}\text { 7-KC induces oxidative stress through } \\
\text { lysosomal dysfunction, aggravating } \\
\text { HASMCs calcification }\end{array}$ \\
\hline
\end{tabular}


Table 1. Cont.

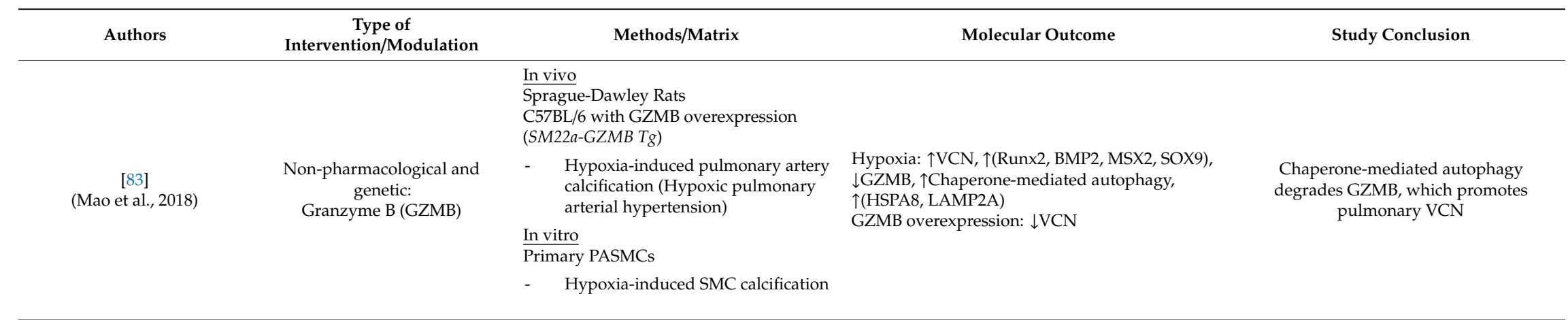

Abbreviations: 3-MA = 3-methyladenine; a-SMA = alpha-smooth muscle actin; AKT = protein kinase B; ALP = Alkaline phosphatase; AMC = Arterial medial calcification; AMPK = AMP-activated protein kinase; ARE = Antioxidant response element; AT-1 = Angiotensin II receptor type 1; BASMC = Bovine aortic smooth muscle cell; BECN = Beclin1; BMP2 = Bone morphogenetic protein 2; BNIP3 = BCL2 and adenovirus E1B 19-kDa-interacting protein 3; CBFA1 = Core-binding factor alpha 1; CKD = Chronic kidney disease; $\mathrm{CRF}=$ Chronic renal failure; Drp1 = Dynamin-1-like protein; ET-B = Endothelin B; Gpat4 = Glycerol-3-phosphate acyltransferase 4; HAP = Hydroxyapatite; HIF-1a = Hypoxia-inducible $\mathrm{CRF}=$ Chronic renal failure; Drp1 = Dynamin-1-like protein; ET-B = Endothelin B; Gpat4 = Glycerol-3-phosphate acyltransferase 4; HAP = Hydroxyapatite; HIF-1a = Hypoxia-inducible
factor 1-alpha; HPD = High Pi diet; HSPA8 = Heat shock protein family A (Hsp70) member 8; JNK = c-Jun N-terminal kinases; LAMP2A = Lysosome associated membrane protein 2; LC3 = Light chain 3; LPA = Lysophosphatidic acid; LSD1 = Lysine-specific histone demethylase 1; MGP = Matrix Gla protein; Msx2 = Msh homeobox 2; mTOR = mammalian target of rapamycin; MV = Matrix vesicle; NR4A1 = Nerve growth factor IB; NRF1 = Nuclear respiratory factor 1; Nrf2 = Nuclear factor erythroid 2-related factor; OC = Osteocalcin; OGT = O-linked N-Acetylglucosamine transferase; OPA1 = Optic atrophy protein 1; OPG = Osteoprotegerin; OPN/OSP = Osteopontin; Ox-LDL = oxidized low density lipoprotein; p-AKT = phosphorylated AKT; p-AMPK = phosphorylated AMPK; p-mTOR = phosphorylated mTOR; -PKA = phosphorylated protein kinase A; p-ULK1 = phosphorylated ULK1; PDK4 = Pyruvate dehydrogenase kinase 4; PGC-1a = peroxisome proliferator-activated receptor gamma coactivator 1-alpha; Pi = inorganic phosphate; PKA = Protein kinase A RUNX2 = Runt-related transcription factor 2; Scd1 $/ 2=$ Stearoyl-CoA desaturase-1/2; SD rats = Sprague Dawley rats; SESN2 = Sestrin 2; $\mathrm{sEV}=$ Small extracellular vesicle; SFA = Saturated fatty acid; Sirt1b = Sirtuin 1; SM22a = smooth muscle protein 22-alpha; SOX9 = SoxE group member SRY (sex determining region Y)- box 9; TFAM = Transcription factor A, mitochondrial; TOMM20/40 = Translocase of the outer mitochondrial membrane 20/40; TRPML1 = Mucolipin-1; UFA = Unsaturated fatty acid; ULK1 = Unc-51 Like Autophagy Activating Kinase 1; $\mathrm{VCN}=$ Vascular calcification; VitD = vitamin D; VSMC = Vascular smooth muscle cell; YAP = Yes-associated protein; $\beta$-GP = $\beta$-glycerophosphate.

Table 2. Studies on autophagy in valvular calcification.

\begin{tabular}{|c|c|c|c|c|}
\hline Authors & Type of Intervention/Modulation & Methods/Matrix & Molecular Outcome & Study Conclusion \\
\hline $\begin{array}{c}{[84]} \\
\text { (Carracedo et al., 2019) }\end{array}$ & $\begin{array}{c}\text { Observational: } \\
\text { Calcific Aortic Valve Stenosis (CAVS) }\end{array}$ & $\begin{array}{l}\frac{\text { ex vivo }}{\text { Aortic valves from patients with CAVS }} \\
\text { In vitro } \\
\text { Primary aortic valvular interstitial } \\
\text { cells (VICs) }\end{array}$ & $\begin{array}{l}\text { CAVS: } \downarrow \text { (ULK1, MAP1LC3A), } \uparrow(B E C N 1, \text { ATG3, } \\
\text { ATG5, ATG7, ATG12), } \downarrow \text { (LAMP1, CTSD), } \\
\uparrow(\text { CTSB, CTSV, CTSL), =TFEB } \\
\text { VICs from CAVS patients: } \uparrow \text { Autophagic flux }\end{array}$ & $\begin{array}{l}\text { Autophagy is upregulated in the aortic } \\
\text { valves from CAVS patients as a } \\
\text { pro-survival mechanism }\end{array}$ \\
\hline
\end{tabular}


Table 2. Cont.

\begin{tabular}{|c|c|c|c|c|}
\hline Authors & Type of Intervention/Modulation & Methods/Matrix & Molecular Outcome & Study Conclusion \\
\hline $\begin{array}{c}\text { [85] } \\
\text { (Deng et al., 2017b) }\end{array}$ & $\begin{array}{c}\text { Observational: } \\
\text { Calcific Aortic Valve Stenosis (CAVS) }\end{array}$ & $\frac{\text { In vitro }}{\text { Primary human aortic VICs }}$ & $\begin{array}{l}\text { VICs from CAVS patients: } \downarrow \text { LC3-II } \\
\text { 3-MA, bafilomycin A1, ATG7 knockdown: } \\
\uparrow \text { Calcification; } \uparrow(\text { BMP2, ALP) } \\
\text { Rapamycin, ATG7 overexpression: } \\
\downarrow \text { Calcification; } \downarrow \text { (BMP2, ALP) }\end{array}$ & $\begin{array}{l}\text { Autophagy attenuates osteochondrogenic } \\
\text { response in human AVICs }\end{array}$ \\
\hline $\begin{array}{c}{[86]} \\
\text { (Somers et al., 2006) }\end{array}$ & $\begin{array}{c}\text { Observational: } \\
\text { Calcific Aortic Valve Stenosis (CAVS) }\end{array}$ & $\frac{\text { Ex vivo }}{\text { Aortic valves from patients with CAVS }}$ & CAVS: $\uparrow$ Ubiquitin & $\begin{array}{l}\text { Large amount of ubiquitinated cells are } \\
\text { present in calcified aortic valve tissue, } \\
\text { indicating possible higher autophagic } \\
\text { activity }\end{array}$ \\
\hline $\begin{array}{c}{[87]} \\
\text { (Bonetti et al., 2017) }\end{array}$ & $\begin{array}{l}\text { Non-pharmacological: } \\
\text { LPS }\end{array}$ & $\begin{array}{l}\frac{\text { In vitro }}{\text { Bovine aortic VICS }} \\
-\quad \mathrm{Pi}(+/- \text { LPS and } 20 \% \text { bovine } \\
\text { macrophage conditioned medium } \\
\quad(\mathrm{CM}))- \text { induced calcification }\end{array}$ & $\begin{array}{l}\text { LPS + CM: } \uparrow \text { Calcification, } \downarrow \text { Autophagic } \\
\text { vacuoles, } \downarrow \text { RER hypertrophy, } \downarrow \text { MAP1LC3 }\end{array}$ & $\begin{array}{l}\text { High Pi concentrations lead to pro-calcific } \\
\text { cell death, while low/middle Pi activate } \\
\text { RER-dependent autophagic activity }\end{array}$ \\
\hline
\end{tabular}




\subsubsection{Stimulating Autophagy in Vascular Calcification by Activating AMPK}

The activation of AMPK with melatonin, metformin, intermedin ${ }_{1-53}$ or MOTS-c ameliorated VCN whereas the AMPK inhibitor compound C worsened VCN. Melatonin ameliorated VSMC calcification in vitro through the activation of AMPK [58]. In line with these findings, metformin ameliorated VSMC calcification in vitro through AMPK activation [59]. Additionally, both the peptide ghrelin and the protein intermedin $1-53\left(\mathrm{IM}_{1-53}\right)$ attenuated VCN through AMPK activation both, in vivo and in vitro [60,61]. Ghrelin induced both LC3-II and Beclin1 while $\mathrm{IM}_{1-53}$ upregulated Sirt1 expression. Also, MOTS-c, a mitochondrial derived peptide, ameliorated VCN in vivo by activating the AMPK pathway [62]. Moreover, compound C (an AMPK inhibitor) abrogated the protective effects of melatonin, metformin and ghrelin on VSMC calcification, as well as their effect on autophagy.

\subsubsection{Stimulation of Autophagy through Unspecified Mechanisms}

A number of studies used experimental interventions that increase autophagy, while the exact molecular mechanisms where not investigated in detail. We have found reports of valproic acid, Polysaccharide from Fuzi (FPS), oestrogen, Nrf2, atorvastatin, miRNA-30b, ANCR that all increased autophagy and ameliorated VCN. Valproic acid, an autophagy inducer, attenuated VCN [51]. In contrast, intermittent administration of valproic acid did not ameliorate VCN [63]. Furthermore, FPS attenuated ox-LDL-induced VSMC calcification in vitro [64]. The authors stated that the protective effects of FPS on VCN are due to its ability to increase autophagy, characterized by an increased LC3-II/LC3-I ratio and decreased p62 expression. In vitro knockdown of Nrf2 impaired autophagy and increased VSMC calcification, while Nrf2 overexpression enhanced the LC3-II/LC3-I ratio and ameliorated VCN [65]. Furthermore, oestrogen inhibited VCN, increased Atg5, LC3-I and LC3-II, and its protective effects were counteracted by 3-MA. The authors therefore stated that the protective effects of oestrogen were autophagy dependent. Likewise, the knockdown of the oestrogen receptor alpha $\left(\mathrm{ER}_{\alpha}\right)$ inhibited autophagy and facilitated VCN [66]. Also, atorvastatin inhibited vascular calcification in vitro, while increasing Atg5, Beclin1 and the LC3-II/LC3-I ratio under calcifying conditions. More so, atorvastatin induced autophagy via the downregulation of the $\beta$-catenin pathway [67]. Furthermore, miRNA-30b attenuated VCN both in vitro and in vivo, whilst increasing both the LC3-II/LC3-I ratio and Beclin1 expression, as well as safeguarding the mitochondrial membrane potential. Therefore, the authors stated that miRNA30b protects against VCN by promoting autophagy through inhibition of Sox9 and mTOR [68]. Moreover, overexpression of anti-differentiation non-coding RNA (ANCR) induced autophagy, characterized by an increase in Atg5, LC3-I and LC3-II, and attenuated VCN both in vitro and in vivo [69]. Klotho knockout mice revealed age-related VCN, while beclin1 overexpression rescued Klotho knockout-induced VCN [70]. Next, iron citrate ameliorated VCN in vitro and enhanced autophagic flux [71]. Last, two studies investigated the effects of advanced glycation end-products (AGEs) on VCN and reported conflicting results. In one study, AGEs increased autophagic flux (demonstrated by an increased number of both autophagosomes and autolysosomes) and promoted VSMC calcification [72]. In another study, AGEs enhanced VCN, however, AGEs were shown to inhibit autophagy through AMPK inhibition and mTOR activation, resulting in decreased Beclin1 and LC3-II levels [73].

\subsubsection{Inhibiting Autophagy in Vascular Calcification}

Several studies reported that inhibition of autophagy with 3-MA, Atg5/7 siRNA, lactate or lysophosphatidic acid (LPA) aggravates VCN. Dai et al. (2013a) showed that inhibiting autophagy with 3-MA or Atg5 knockdown enhances VCN in vitro. Other studies confirmed that 3-MA aggravates VSMC calcification $[52,53,72]$. Furthermore, multiple studies added 3-MA, in combination with an autophagy, inducing pharmacological intervention, thereby showing that 3-MA abolishes the beneficial effects of autophagy induction on VCN $[59,61,64,66,67]$. Additionally, CD137 activation led to JNK-mediated disrupted autophagic flux and aggravated VCN [74]. Furthermore, O-GlcNAc 
transferase (OGT) was upregulated in CKD-associated VCN, thereby, enhancing Yes-associated protein (YAP) glycosylation. The authors stated that elevated or in vitro overexpression of (glycosylated) YAP inhibits autophagic activity, facilitating VCN [75]. Next, excess amounts of saturated lysophosphatidic acid (LPA), a saturated fatty acid, were shown to inhibit the early steps of autophagosome formation, thereby, blocking autophagic flux. Moreover, saturated LPAs facilitated VCN while unsaturated LPAs counteracted these effects and restored autophagic activity [76]. Alongside general autophagy, mitophagy plays a role in VCN. Lactate aggravated VSMC calcification in vitro by inhibiting BCL2 and adenovirus E1B 19-kDa-interacting protein 3 (BNIP3)-mediated mitophagy, leading to mitochondrial dysfunction $[77,78]$.

\subsection{Lysosomal Function in Vascular Calcification}

Impairment of lysosomal function has been shown to aggravate VCN. We have found a limited number of studies that interfered with lysosomal function by: (1) Deletion of Mcoln1 or Asah1 genes, (2) overexpression of the Smpd1 gene, or (3) administration of 7-ketocholesterol (7-KC) aggravated VCN. However, lysosomal function was also shown to promote VCN by degrading the anti-calcifying protein granzyme B. Knocking out Mcoln1 enhanced VCN both, in vivo and in vitro by impairing lysosomal trafficking, and consequentially enhancing secretion of pro-calcific small extracellular vesicles (sEVs) (Table 1) [79]. Asah1 gene deletion also aggravated VCN and decreased TRPML1 activity, leading to impaired lysosomal trafficking and enhanced pro-calcific sEV secretion [80]. Additionally, sphingomyelin phosphodiesterase 1 (Smpd1) overexpression lead to increased VCN, impaired lysosomal trafficking, enhanced secretion of sEVs and increased arterial stiffness [81]. 7-KC was shown to aggravate VCN by inducing lysosomal dysfunction and autophagosome accumulation [82] Lastly, granzyme B was degraded in hypoxia-induced pulmonary calcification by chaperone-mediated autophagy, characterized by an increase in Heat Shock Protein Family A (Hsp70) Member 8 (HSPA8) and lysosome-associated membrane protein 2 (LAMP2A). Conversely, granzyme B overexpression alleviated VCN both in vivo and in vitro [83].

\subsection{Autophagy in Valvular Calcification}

Three studies used aortic valves from human patients, obtained after replacement surgery, to investigate the role of autophagy in AVCN (Table 2). In one study [84], ULK1 and MAP1LC3A were decreased, while BECN1, ATG3, ATG5, ATG7 and ATG12 were increased in the aortic valves from patients with calcific aortic valve stenosis (CAVS). Additionally, LAMP1 and cathepsin D (CTSD) were reduced, while an increase was observed in cathepsin B (CTSB), V (CTSV) and L (CTSL) in these patients. TFEB expression was unchanged. Further, the authors observed increased autophagic flux in VICs, isolated from the aortic valves from patients with CAVS. In contrast, another study [85] reported lower LC3-II expression in VICs from patients with CAVS. Additionally, 3-MA, bafilomycin A1 and ATG7 knockdown all increased BMP2 and alkaline phosphatase (ALP) levels, indicating enhanced VIC calcification. Moreover, ATG7 overexpression or rapamycin alleviated VIC calcification. A histological evaluation of aortic valves from patients with CAVS revealed an increased amount of ubiquitin labelling in calcified tissue. Therefore, the authors [86] stated that autophagic activity might be enhanced in these tissues. Finally, in the last study [87], LPS was combined with conditioned medium of bovine macrophages to enhance the effect of Pi-induced bovine VIC calcification. At high Pi concentrations, MAP1LC3 expression, as well as the total number of autophagic vacuoles decreased, whereas low-to-middle concentrations of Pi resulted in rough endoplasmic reticulum (RER)-dependent autophagic activity [87]. 


\section{Discussion}

\subsection{Inhibiting mTOR Protects against Vascular Calcification}

Nine studies investigated the effect of AMPK or mTOR (or both) on vascular calcification (VCN) (Figure 3). One study (Zhan et al., 2014) reported increased mTOR activity in in vitro calcification and another study (Zhao et al., 2015a) found similar results in vivo. Moreover, mTOR overexpression has been shown to enhance VCN, while mTOR inhibition ameliorated VSMC calcification. Consequentially, rapamycin, adiponectin and bavachin have been shown to attenuate VCN due to their ability to inhibit mTOR activity [54,56,57]. Epigenetic regulation of mTOR activity has also been shown to modulate VCN. Under pro-calcifying conditions, HDAC1 and LSD1 expression were decreased, and increased, respectively [52]. LSD1 is implicated in various biological processes, such as cell proliferation, stem cell differentiation and enhancing mTORC1 activity [88]. Increasing HDAC1 activity or silencing LSD1 decreased mTOR activity and mitigated VCN [52]. These results indicate that mTOR plays an important role in the pathogenesis of VCN. A possible mechanism as to the reasons why mTOR contributes to the development of VCN, is via mTOR-mediated inhibition of Klotho. Notably, the Klotho protein, originally identified as an aging suppressor, possesses strong anti-calcification properties and prevents osteogenic trans differentiation of VSMCs [89]. One study [56] demonstrated that mTOR (over)expression inhibits Klotho expression whereas rapamycin alleviates this repression and ameliorates VSMC calcification. Interestingly, intermedin ${ }_{1-53}\left(\mathrm{IM}_{1-53}\right)$ also ameliorated VCN while the authors additionally observed a marked increase in Klotho expression after $\mathrm{IM}_{1-53}$ treatment. This increase in Klotho expression by $\mathrm{IM}_{1-53}$, is possibly the consequence of AMPK-dependent inhibition of mTOR [60]. Indeed, there are multiple lines of evidence supporting AMPK as an anti-VSMC calcification mechanism. Melatonin, metformin, ghrelin, intermedin $1-53$ and MOTS-c have all been demonstrated to ameliorate VCN in vitro and/or in vivo, accredited due to their ability to increase AMPK activity [58-62].

Collectively, these studies indicate that mTOR inhibition, either directly or resulting from AMPK activation, ameliorates VCN and prevents an osteochondrogenic phenotype switch of VSMCs. Whether mTOR modulates VCN through autophagy inhibition is less certain. mTOR has also been shown to enhance Runx2 expression by modulating the oestrogen receptor alpha [90] in addition to suppressing the anti-calcification protein Klotho. Moreover, mTOR plays an important role in skeletal development, facilitating the progression of the preosteoblast stage to the mature osteoblast stage [91]. Besides the suppression of autophagy, mTOR has multiple other functions such as inhibition of cell growth and protein synthesis [92]. Hence, the protective effects of mTOR inhibition in VCN are generally accepted, these effects might partially be unrelated to autophagy.

\subsection{Autophagy Protects against Vascular Calcification}

While, studies targeting mTOR in pro-calcifying conditions do not unambiguously prove the role of autophagy in VCN, inhibition of autophagy by 3-MA or by Atg5 silencing consistently increased VCN [51-53,72]. Also, other interventions inhibiting autophagy, such as CD137 activation, lead to aggravated VCN [74]. Furthermore, in pro-calcifying conditions, OGT is upregulated, enhancing YAP glycosylation. YAP glycosylation inhibits autophagy while YAP silencing ameliorates VCN [75]. $\mathrm{Xu}$ et al. proposed that YAP inhibits autophagy through activation of MST1 [75]. However, only the LC3-II/LC3-I ratio and p62 levels were measured, which is not sufficient to prove the effect of YAP on autophagic flux. Furthermore, another study demonstrated that saturated fatty acids (SFAs), which are increased in patients with CKD, inhibited autophagic flux drastically and facilitated VCN [76]. Collectively, these data demonstrate that autophagy is protective in VCN. 


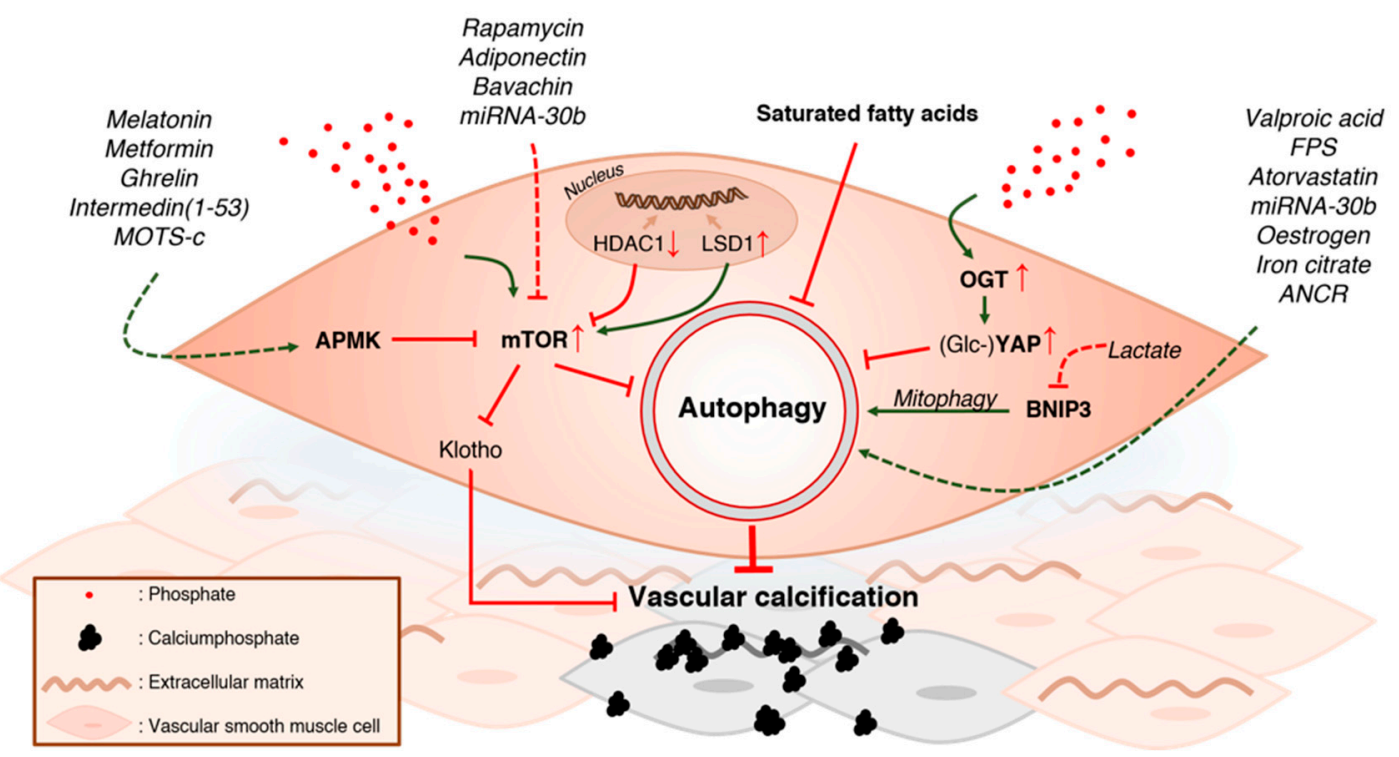

Figure 3. Modulating the autophagic machinery in vascular calcification. A high Pi environment promotes vascular smooth muscle cell (VSMC) calcification. This pro-calcific environment increases the activity of the mammalian target of rapamycin (mTOR), as well as O-GlcNAc transferase (OGT). The latter enhances YAP glycosylation. Additionally, epigenetic regulation of mTOR activity is also affected by the high Pi environment. Histone deacetylase 1 (HDAC1) is downregulated, while lysine-specific histone demethylase A1 (LSD1) is upregulated, resulting in enhanced mTOR activity. Both the increase in glycosylated (Glc) YAP and mTOR activity inhibit autophagy, facilitating VSMC calcification. Therefore, direct or AMPK-mediated inhibition of mTOR, through the use of different pharmacological agents, alleviates mTOR-dependent suppression of autophagy and ameliorates VSMC calcification. Saturated fatty acids, which are present in the pro-calcific environment, as seen in patients with chronic kidney disease, inhibit autophagy and are shown to facilitate VSMC calcification. Stimulation of autophagy with various pharmacological agents has been shown to attenuate VSMC calcification. Lastly, BNIP3-mediated mitophagy plays an important role in VSMC calcification, as inhibition of this pathway with lactate, promotes mineralization. AMPK = AMP-activated kinase, $\mathrm{mTOR}=$ mammalian target of rapamycin, HDAC1 $=$ Histone deacetylase 1, LSD1 $=$ Lysine-specific histone demethylase A1, OGT = O-GlcNAc transferase, Glc = Glycosylated, YAP = Yes-associated protein, BNIP3 = BCL2 and adenovirus E1B 19-kDa-interacting protein 3, ANCR = anti-differentiation non-coding RNA.

A few studies have investigated the role of mitophagy in VCN. Lactate was shown to inhibit BNIP3-mediated mitophagy, worsening VSMC calcification $[77,78]$. In contrast, metformin ameliorated VCN by inducing mitochondrial biogenesis through mitophagy [59]. Additionally, miRNA-30b ameliorated VSMC calcification and rescued mitochondrial membrane potential under calcifying conditions [68]. It is known that high Pi levels induce mitochondrial defects [93]. While, it can be expected that mitophagy plays a crucial role in mitigating aggravated oxidative stress, due to mitochondrial dysfunction [94], more research is warranted.

Importantly, many studies used LC3-II/LC3-I ratios for evaluating autophagic flux. However, this ratio is rather unreliable and current guidelines on quantification of autophagic flux do not recommend the use of LC3-II/LC3-I ratios as a sole predictor of autophagic flux [95]. Furthermore, oestrogen, ANCR and atorvastatin have been demonstrated to enhance Atg5 expression and miRNA-30b has been shown to induce Beclin1 [66-69]. Only one study [71] determined autophagic flux (i.e., the turnover of LC3-II) by using an autophagy inhibitor, as is indicated in the most recent guidelines on the evaluation of autophagy [95]. Exemplary of the importance of using appropriate methodologies for evaluating autophagic flux are two studies investigating the effect of AGEs. While both studies confirmed that AGEs worsen VSMC calcification, conflicting conclusions with respect to autophagy 
induction were made. Liu et al. (2020b) stated that AGEs inhibit autophagy while Yang et al. (2019) reported increased autophagic flux. However, Liu et al. (2020b) only investigated autophagy indirectly, finding AMPK inhibition, activation of mTOR activity and decreased Beclin1 and LC3-II levels. In contrast, Yang et al. (2019) used mRFP-GFP-LC3 double labelling, which is a convenient and reliable method for monitoring autophagic flux, as it enables the simultaneous evaluation of autophagosomes (GFP-RFP) and autolysosomes (RFP) [95]. Moreover, the two research groups (Liu et al. and Yang et al.) used slightly different conditions, which could also explain their contrasting results. Nonetheless, these contradicting results emphasize the need for a more standardized approach for assessing autophagic activity.

In general, inducing autophagy with pharmacological agents ameliorates VSMC calcification. While the selectivity of pharmacological agents used to induce autophagy is often limited, experimental studies with knockout of specific autophagy genes showed deterioration of VSMC calcification. Likewise, overexpression of Beclin1 protected against VCN. Therefore, there is a consensus that autophagy is protective in VCN and may be an attractive therapeutic target in VCN.

\subsection{Intact Lysosomal Function Is Important for Controlling Vascular Calcification}

While, hydroxyapatite itself damages cells and impairs lysosomes, multiple studies examined how impairing lysosomal function affects VCN (Figure 4) [55]. The TRPML1 channel mediates the fusion of lysosomes with autophagosomes. Notably, TRPML1 also regulates autophagy by regulating TFEB and by activating both calcium/calmodulin-dependent protein kinase kinase (CaMKK $\beta)$-mediated signalling and ULK1 and hVPS34 [96]. Knocking-out Mcoln1, which is the gene that encodes TRPML1, resulted in increased VCN both in vitro and in vivo. Moreover, increased secretion of pro-calcific sEVs was reported in the absence of TRPML1 [79]. sEVs contain a variety of cargoes such as proteins and lipids that can chelate Pi and calcium. These calcium Pi-loaded vesicles tend to aggregate and form microcalcifications, acting as nucleation points and facilitating VCN $[97,98]$. sEVs are generated through different mechanisms, such as the fusion of multivesicular bodies (MVBs) with the plasma membrane or by budding, and consequent fission, of the plasma membrane [99]. Importantly, there is an inverse relation between the pathways that degrade MVBs and those that secrete sEVs. Indeed, it has been shown that inhibition of lysosomal degradation, enhances the release of sEVs [100]. Similarly, knocking out Asah1, the gene encoding for acid ceramidase (AC), enhanced sEV secretion and VCN. AC metabolizes ceramide into sphingosine (Sph) in lysosomes. Sph is crucial for proper functioning of the TRPML1 channel. A lack of AC impairs lysosome trafficking, leading to MVB accumulation and sEV release [101]. Last, the overexpression of lysosomal acid sphingomyelinase (ASM), encoded by Smpd1, aggravated calcification and also enhanced sEVs secretion [81]. An imbalance between ceramide production by ASM through sphingomyelin and ceramide consumption by AC, causes defective lysosomal acidification. Defective lysosomal acidification disrupts the function of TRPML1 and therefore impairs lysosomal trafficking [101,102]. Hence, sphingolipid metabolism is crucial for proper lysosome function and any defects in this pathway lead to aggravated VCN.

Granzyme B, a cytotoxic serum protease protein, was demonstrated to mitigate hypoxia-induced pulmonary arterial calcification (PAC) [83]. However, during hypoxia, granzyme B was degraded by chaperone-mediated autophagy (CMA). CMA is a proteolytic systems where intracellular proteins are degraded by lysosomes. CMA substrates are selectively targeted to the lysosome by the actions of chaperones and lysosomal membrane proteins such as LAMP1 [103]. Overexpression of granzyme B in PASMCs counteracted hypoxia-induced calcification [83]. This finding suggests that granzyme B plays a protective role in hypoxia induced PASMC calcification, whereas CMA aggravates calcification through granzyme B degradation. 


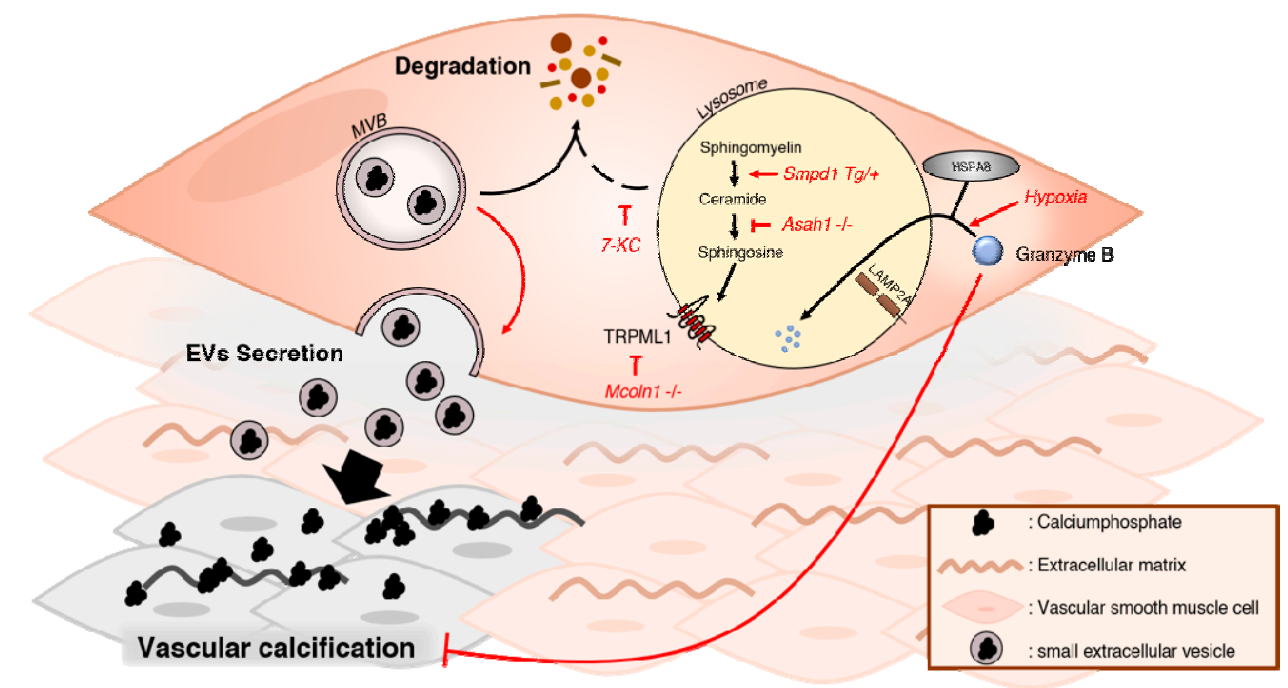

Figure 4. Modulating lysosomal function in vascular calcification. In a pro-calcific environment, calciumphosphate-loaded multivesicular bodies (MVBs), a type of late endosome, can either be degraded or secrete their contents to the extracellular matrix. In physiological conditions, there is a balance between degradation and secretion. Impairment of lysosomal function leads to decreased degradation of MVBs and enhanced secretion of pro-calcific extracellular vesicles (EVs). Transient receptor potential mucolipin 1 (TRPML1) is important for proper lysosome functioning, mediating the fusion of lysosomes with autophagosomes and endosomes. Knockout of the TRPML1 channel through the deletion of the mucolipin 1 gene, Mcoln1, impairs lysosome function, thereby enhancing the release of pro-calcific EVs and aggravating vascular calcification (VCN). Sphingosine is necessary for TRPML1 activity. Impairing the biosynthesis of sphingosine from ceramide, through the inhibition of the enzyme acid ceramidase, consequently impairs TRPML1 function, leading to enhanced sEV secretion and VCN. The balance between ceramide and sphingosine levels inside the lysosome dictates lysosomal function. Enhanced levels of ceramide through overexpression of the Smpd1 gene, which encodes for acid sphingomyelinase, impairs lysosome function. Overexpression of Smpd1 leads to enhanced secretion of EVs and VCN. Additionally, impairing lysosomal function with 7-ketocholesterol aggravates VCN. Finally, in a hypoxic environment, granzyme B is degraded via Heat shock protein family A (Hsp70) member 8 (HSPA8)-dependent chaperone-mediated autophagy. However, granzyme B has been shown to be protective against VCN. Therefore, chaperone-mediated autophagy worsens hypoxia-induced calcification. $\mathrm{sEV}=$ small Extracellular vesicle, $\mathrm{MVB}=$ Multivesicular bodies, HSPA8 = Heat shock protein family A (Hsp70) member 8.

Finally, 7-KC was shown to induce lysosomal dysfunction and oxidative stress, thereby aggravating $\mathrm{VCN}$ [82]. It is known that oxysterols such as 7-KC accumulate in the lysosomes, diminishing lysosomal proteolytic enzyme activity and resulting in inhibition of the fusion between lysosomes with autophagosomes or with endosomes [82]. Hence, both the autophagic machinery and the degradation of EVs are impaired, potentially attributing to the development of VCN.

Taken together, there is compelling evidence that lysosomal function plays a critical role in mitigating VCN through inhibition of sEV secretion and reducing oxidative stress. Therefore, enhancing lysosomal function or lysosomal biogenesis could be a promising strategy for ameliorating VCN.

\subsection{Protective Autophagy Is Upregulated in Aortic Valve Calcification}

Aortic valve calcification (AVCN) is the major cause of valvular stenosis and shares many similarities to VCN, such as the transition of VICs to osteoblast-like cells and the formation of pro-calcific MVs [104]. However, little is known about modulating autophagy in valvular calcification. Three studies investigated aortic valves from patients with calcific aortic valve stenosis (CAVS) and all three studies found signs of enhanced autophagic flux. In one study [84], an upregulation of ATGs 
proteins was observed, as well as an increase in expression of lysosomal proteases. Also in vitro, using the LC3-II turnover assay, autophagic flux was enhanced in VICs [84]. In line with these findings, Somers et al. (2006) discovered more ubiquitin labelling in calcified tissue, possibly indicating enhanced autophagic activity. However, ubiquitin is not exclusive to the autophagy pathway, acting as a signal molecule for the ubiquitin-proteasome system as well [105]. Therefore, looking solely at ubiquitin does not provide sound evidence for claiming altered autophagic activity. Furthermore, inhibition of autophagy, either pharmacologically or genetically, aggravated VIC calcification [85]. Interestingly, the concentration of Pi seems to determine the type of autophagy during VIC calcification. Low-to-middle concentrations of Pi led to RER-dependent autophagy, whereas high doses drastically decreased the total number of autophagic vacuoles and MAP1LC3 [87]. All these findings indicated that, as in $\mathrm{VCN}$, autophagy is upregulated and acts as a protective mechanism. However, more research is needed to gain more insight in how autophagy or lysosomal function is affected in valvular calcification and whether its upregulation or rescue is beneficial.

\subsection{Limitations}

A notable weakness of this review is the heterogeneity between the studies. There are differences between in vivo and in vitro models. Furthermore, within in vitro studies there is considerable variation in the concentration of minerals, ranging from $1.4-5 \mathrm{mM}$ for Pi (1.25-10 $\mathrm{mM}$ for $\beta-\mathrm{GP})$ and $1.4-3 \mathrm{mM}$ for calcium, which do not reflect physiological conditions. These experimental conditions should be taken into account when comparing the results, especially in the context of autophagy. Moreover, autophagy research is hampered by a lack of standardized methods for evaluating autophagy in different experimental settings. While guidelines for appropriate methods for investigating autophagy have been published (and are regularly updated), it became apparent that standardization of methods has not been achieved yet. Due to the large heterogeneity in models and methodologies, no meta-analysis was conducted. Finally, no quality assessment of the included studies was performed, given that the appropriate tools could not be found for this type of primary research.

\section{Concluding Remarks and Future Perspectives}

Most studies (97\%) reported a protective effect of autophagy and lysosomal function in VCN. Only one study [83] reported dissonant results. Pharmacological induction of autophagy was most often achieved through mTOR inhibition (directly or indirectly via AMPK activation) and consistently ameliorated VCN. However, the selectivity of current autophagy inducing pharmacological compounds is not absolute. Therefore, the development of novel, more selective autophagy inducers is crucial to realize the full therapeutic potential in VCN. Additionally, several recent studies indicate that lysosomes are crucial for mitigating VCN. Moreover, impaired lysosomal function aggravated VSMC calcification, but studies focusing on improving lysosomal function in $\mathrm{VCN}$ were not found. Future research is required to fully understand the role of lysosomal function in VCN and its possible synergy with autophagy. Finally, there is evidence that autophagy acts as a protective mechanism in AVCN as well. Yet, the data regarding this topic is sparse and more research is warranted. Besides, several methodological limitations still need to be overcome. Since autophagy is a dynamic and complex process, it is advised that future studies focus on measuring autophagic flux, with a view to standardisation.

Author Contributions: C.H.G.N. and J.O.H. were responsible for the article selection process, data extraction and bias assessment. Any disagreements were discussed together with the other authors. C.H.G.N. drafted the manuscript. J.O.H., W.M., G.R.Y.D.M. and P.-J.G. critically reviewed and edited the manuscript. All authors have read and approved the final version of the manuscript.

Funding: This work was supported by the Fund for scientific Research (FWO)-Flanders (G041216N) and the University of Antwerp (GOA 33931, DOCPRO 36663, BOF SEP 41477, BOF SEP 41478). C.H.G.N. is a fellow of the FWO-Flanders (grant $\mathrm{N}^{\circ} 1 \mathrm{~S} 24720 \mathrm{~N}$ ).

Acknowledgments: The authors are grateful to Bronwen Martin for critical reading of the manuscript. 
Conflicts of Interest: The funders had no role in the design of the study; in the collection, analyses, or interpretation of data; in the writing of the manuscript, or in the decision to publish the results.

\section{References}

1. Yin, Z.; Pascual, C.; Klionsky, D.J. Autophagy: Machinery and regulation. Microb. Cell 2016, 3, 588-596. [CrossRef] [PubMed]

2. Parzych, K.R.; Klionsky, D.J. An overview of autophagy: Morphology, mechanism, and regulation. Antioxid. Redox Signal. 2014, 20, 460-473. [CrossRef] [PubMed]

3. Glick, D.; Barth, S.; Macleod, K.F. Autophagy: Cellular and molecular mechanisms. J. Pathol. 2010, $221,3-12$. [CrossRef]

4. Seranova, E.; Connolly, K.J.; Zatyka, M.; Rosenstock, T.R.; Barrett, T.; Tuxworth, R.I.; Sarkar, S. Dysregulation of autophagy as a common mechanism in lysosomal storage diseases. Essays Biochem. 2017, 61, 733-749. [CrossRef] [PubMed]

5. Das, G.; Shravage, B.V.; Baehrecke, E.H. Regulation and function of autophagy during cell survival and cell death. Cold Spring Harb. Perspect. Biol. 2012, 4. [CrossRef]

6. Yorimitsu, T.; Klionsky, D.J. Autophagy: Molecular machinery for self-eating. Cell Death Differ. 2005, 12, 1542-1552. [CrossRef]

7. Kim, Y.C.; Guan, K.L. mTOR: A pharmacologic target for autophagy regulation. J. Clin. Investig. 2015, 125, 25-32. [CrossRef]

8. Hardie, D.G. AMPK and autophagy get connected. EMBO J. 2011, 30, 634-635. [CrossRef]

9. Jang, M.; Park, R.; Kim, H.; Namkoong, S.; Jo, D.; Huh, Y.H.; Jang, I.S.; Lee, J.I.; Park, J. AMPK contributes to autophagosome maturation and lysosomal fusion. Sci. Rep. 2018, 8, 12637. [CrossRef]

10. Periyasamy-Thandavan, S.; Jiang, M.; Schoenlein, P.; Dong, Z. Autophagy: Molecular machinery, regulation, and implications for renal pathophysiology. Am. J. Physiol. Ren. Physiol. 2009, 297, F244-F256. [CrossRef]

11. De Munck, D.G.; De Meyer, G.R.; Martinet, W. Autophagy as an emerging therapeutic target for age-related vascular pathologies. Expert Opin. Targets 2020, 24, 131-145. [CrossRef] [PubMed]

12. Dikic, I.; Elazar, Z. Mechanism and medical implications of mammalian autophagy. Nat. Rev. Mol. Cell Biol. 2018, 19, 349-364. [CrossRef] [PubMed]

13. Martinez-Lopez, N.; Athonvarangkul, D.; Singh, R. Autophagy and aging. Adv. Exp. Med. Biol. 2015, 847, 73-87. [CrossRef]

14. Schiattarella, G.G.; Hill, J.A. Therapeutic targeting of autophagy in cardiovascular disease. J. Mol. Cell Cardiol. 2016, 95, 86-93. [CrossRef] [PubMed]

15. Abdellatif, M.; Sedej, S.; Carmona-Gutierrez, D.; Madeo, F.; Kroemer, G. Autophagy in Cardiovascular Aging. Circ. Res. 2018, 123, 803-824. [CrossRef] [PubMed]

16. Martinet, W.; Knaapen, M.W.; Kockx, M.M.; De Meyer, G.R. Autophagy in cardiovascular disease. Trends Mol. Med. 2007, 13, 482-491. [CrossRef] [PubMed]

17. Greenwald, S.E. Ageing of the conduit arteries. J. Pathol. 2007, 211, 157-172. [CrossRef] [PubMed]

18. Hu, M.C.; Shi, M.; Zhang, J.; Quiñones, H.; Griffith, C.; Kuro-o, M.; Moe, O.W. Klotho deficiency causes vascular calcification in chronic kidney disease. J. Am. Soc. Nephrol. 2011, 22, 124-136. [CrossRef]

19. Neven, E.; De Schutter, T.M.; De Broe, M.E.; D'Haese, P.C. Cell biological and physicochemical aspects of arterial calcification. Kidney Int. 2011, 79, 1166-1177. [CrossRef]

20. Shao, J.S.; Cheng, S.L.; Sadhu, J.; Towler, D.A. Inflammation and the osteogenic regulation of vascular calcification: A review and perspective. Hypertension 2010, 55, 579-592. [CrossRef]

21. Hofmann Bowman, M.A.; McNally, E.M. Genetic pathways of vascular calcification. Trends Cardiovasc. Med. 2012, 22, 93-98. [CrossRef] [PubMed]

22. Cannata-Andia, J.B.; Roman-Garcia, P.; Hruska, K. The connections between vascular calcification and bone health. Nephrol. Dial. Transpl. 2011, 26, 3429-3436. [CrossRef] [PubMed]

23. Jono, S.; McKee, M.D.; Murry, C.E.; Shioi, A.; Nishizawa, Y.; Mori, K.; Morii, H.; Giachelli, C.M. Phosphate regulation of vascular smooth muscle cell calcification. Circ. Res. 2000, 87, E10-E17. [CrossRef] [PubMed]

24. Blumenthal, H.T.; Lansing, A.I.; Wheeler, P.A. Calcification of the Media of the Human Aorta and Its Relation to Intimal Arteriosclerosis, Ageing and Disease. Am. J. Pathol. 1944, 20, 665-687. 
25. Evrard, S.; Delanaye, P.; Kamel, S.; Cristol, J.P.; Cavalier, E. Vascular calcification: From pathophysiology to biomarkers. Clin. Chim. Acta 2015, 438, 401-414. [CrossRef] [PubMed]

26. Giachelli, C.M. Vascular calcification mechanisms. J. Am. Soc. Nephrol. 2004, 15, 2959-2964. [CrossRef]

27. Moe, S.M.; Duan, D.; Doehle, B.P.; O'Neill, K.D.; Chen, N.X. Uremia induces the osteoblast differentiation factor Cbfa1 in human blood vessels. Kidney Int. 2003, 63, 1003-1011. [CrossRef]

28. Leopold, J.A. Vascular calcification: Mechanisms of vascular smooth muscle cell calcification. Trends Cardiovasc. Med. 2015, 25, 267-274. [CrossRef]

29. Steitz, S.A.; Speer, M.Y.; Curinga, G.; Yang, H.Y.; Haynes, P.; Aebersold, R.; Schinke, T.; Karsenty, G.; Giachelli, C.M. Smooth muscle cell phenotypic transition associated with calcification: Upregulation of Cbfa1 and downregulation of smooth muscle lineage markers. Circ. Res. 2001, 89, 1147-1154. [CrossRef]

30. Kapustin, A.N.; Shanahan, C.M. Emerging roles for vascular smooth muscle cell exosomes in calcification and coagulation. J. Physiol. 2016, 594, 2905-2914. [CrossRef]

31. Mizobuchi, M.; Towler, D.; Slatopolsky, E. Vascular calcification: The killer of patients with chronic kidney disease. J. Am. Soc. Nephrol. 2009, 20, 1453-1464. [CrossRef] [PubMed]

32. Giachelli, C.M.; Bae, N.; Almeida, M.; Denhardt, D.T.; Alpers, C.E.; Schwartz, S.M. Osteopontin is elevated during neointima formation in rat arteries and is a novel component of human atherosclerotic plaques. J. Clin. Investig. 1993, 92, 1686-1696. [CrossRef] [PubMed]

33. Levy, R.J.; Schoen, F.J.; Levy, J.T.; Nelson, A.C.; Howard, S.L.; Oshry, L.J. Biologic determinants of dystrophic calcification and osteocalcin deposition in glutaraldehyde-preserved porcine aortic valve leaflets implanted subcutaneously in rats. Am. J. Pathol. 1983, 113, 143-155. [PubMed]

34. Reynolds, J.L.; Joannides, A.J.; Skepper, J.N.; McNair, R.; Schurgers, L.J.; Proudfoot, D.; Jahnen-Dechent, W.; Weissberg, P.L.; Shanahan, C.M. Human vascular smooth muscle cells undergo vesicle-mediated calcification in response to changes in extracellular calcium and phosphate concentrations: A potential mechanism for accelerated vascular calcification in ESRD. J. Am. Soc. Nephrol. 2004, 15, 2857-2867. [CrossRef] [PubMed]

35. Lacolley, P.; Regnault, V.; Segers, P.; Laurent, S. Vascular Smooth Muscle Cells and Arterial Stiffening: Relevance in Development, Aging, and Disease. Physiol. Rev. 2017, 97, 1555-1617. [CrossRef]

36. Bragdon, B.; Moseychuk, O.; Saldanha, S.; King, D.; Julian, J.; Nohe, A. Bone morphogenetic proteins: A critical review. Cell Signal 2011, 23, 609-620. [CrossRef]

37. Chen, G.; Deng, C.; Li, Y.P. TGF- $\beta$ and BMP signaling in osteoblast differentiation and bone formation. Int. J. Biol. Sci. 2012, 8, 272-288. [CrossRef]

38. Cai, J.; Pardali, E.; Sánchez-Duffhues, G.; ten Dijke, P. BMP signaling in vascular diseases. FEBS Lett. 2012, 586, 1993-2002. [CrossRef]

39. Asahina, I.; Sampath, T.K.; Nishimura, I.; Hauschka, P.V. Human osteogenic protein-1 induces both chondroblastic and osteoblastic differentiation of osteoprogenitor cells derived from newborn rat calvaria. J. Cell Biol. 1993, 123, 921-933. [CrossRef]

40. Kang, Q.; Sun, M.H.; Cheng, H.; Peng, Y.; Montag, A.G.; Deyrup, A.T.; Jiang, W.; Luu, H.H.; Luo, J.; Szatkowski, J.P.; et al. Characterization of the distinct orthotopic bone-forming activity of 14 BMPs using recombinant adenovirus-mediated gene delivery. Gene 2004, 11, 1312-1320. [CrossRef]

41. Hruska, K.A.; Mathew, S.; Saab, G. Bone morphogenetic proteins in vascular calcification. Circ. Res. 2005, 97, 105-114. [CrossRef] [PubMed]

42. He, F.; Wang, H.; Ren, W.Y.; Ma, Y.; Liao, Y.P.; Zhu, J.H.; Cui, J.; Deng, Z.L.; Su, Y.X.; Gan, H.; et al. BMP9/COX-2 axial mediates high phosphate-induced calcification in vascular smooth muscle cells via Wnt/ $\beta$-catenin pathway. J. Cell Biochem. 2018, 119, 2851-2863. [CrossRef] [PubMed]

43. Li, X.; Yang, H.Y.; Giachelli, C.M. BMP-2 promotes phosphate uptake, phenotypic modulation, and calcification of human vascular smooth muscle cells. Atherosclerosis 2008, 199, 271-277. [CrossRef] [PubMed]

44. Davenport, C.; Harper, E.; Forde, H.; Rochfort, K.D.; Murphy, R.P.; Smith, D.; Cummins, P.M. RANKL promotes osteoblastic activity in vascular smooth muscle cells by upregulating endothelial BMP-2 release. Int. J. Biochem. Cell Biol. 2016, 77, 171-180. [CrossRef]

45. Lee, K.M.; Kang, H.A.; Park, M.; Lee, H.Y.; Choi, H.R.; Yun, C.H.; Oh, J.W.; Kang, H.S. Interleukin-24 attenuates $\beta$-glycerophosphate-induced calcification of vascular smooth muscle cells by inhibiting apoptosis, the expression of calcification and osteoblastic markers, and the Wnt/ $\beta$-catenin pathway. Biochem. Biophys. Res. Commun. 2012, 428, 50-55. [CrossRef] 
46. Rong, S.; Zhao, X.; Jin, X.; Zhang, Z.; Chen, L.; Zhu, Y.; Yuan, W. Vascular calcification in chronic kidney disease is induced by bone morphogenetic protein- 2 via a mechanism involving the $\mathrm{Wnt} / \beta$-catenin pathway. Cell Physiol. Biochem. 2014, 34, 2049-2060. [CrossRef]

47. Phadwal, K.; Feng, D.; Zhu, D.; MacRae, V.E. Autophagy as a novel therapeutic target in vascular calcification. Pharmacol. Ther. 2020, 206, 107430. [CrossRef]

48. Pescatore, L.A.; Gamarra, L.F.; Liberman, M. Multifaceted Mechanisms of Vascular Calcification in Aging. Arter. Thromb. Vasc. Biol. 2019, 39, 1307-1316. [CrossRef]

49. Shanahan, C.M. Autophagy and matrix vesicles: New partners in vascular calcification. Kidney Int. 2013, 83, 984-986. [CrossRef]

50. Liberati, A.; Altman, D.G.; Tetzlaff, J.; Mulrow, C.; Gøtzsche, P.C.; Ioannidis, J.P.; Clarke, M.; Devereaux, P.J.; Kleijnen, J.; Moher, D. The PRISMA statement for reporting systematic reviews and meta-analyses of studies that evaluate health care interventions: Explanation and elaboration. PLoS Med. 2009, 6, e1000100. [CrossRef]

51. Dai, X.-Y.; Zhao, M.-M.; Cai, Y.; Guan, Q.-C.; Zhao, Y.; Guan, Y.; Kong, W.; Zhu, W.-G.; Xu, M.-J.; Wang, X. Phosphate-induced autophagy counteracts vascular calcification by reducing matrix vesicle release. Kidney Int. 2013, 83, 1042-1051. [CrossRef] [PubMed]

52. Zhou, J.; Zhou, H.; Liu, C.; Huang, L.; Lu, D.; Gao, C. HDAC1-mediated deacetylation of LSD1 regulates vascular calcification by promoting autophagy in chronic renal failure. J. Cell. Mol. Med. 2020, 24, 8636-8649. [CrossRef] [PubMed]

53. Frauscher, B.; Kirsch, A.H.; Schabhuttl, C.; Schweighofer, K.; Ketszeri, M.; Pollheimer, M.; Dragun, D.; Schroder, K.; Rosenkranz, A.R.; Eller, K.; et al. Autophagy Protects From Uremic Vascular Media Calcification. Front. Immunol. 2018, 9, 1866. [CrossRef] [PubMed]

54. Zhan, J.K.; Wang, Y.J.; Wang, Y.; Wang, S.; Tan, P.; Huang, W.; Liu, Y.S. The mammalian target of rapamycin signalling pathway is involved in osteoblastic differentiation of vascular smooth muscle cells. Can. J. Cardiol. 2014, 30, 568-575. [CrossRef] [PubMed]

55. Liu, H.; Huang, L.H.; Sun, X.Y.; Ouyang, J.M. High-phosphorus environment promotes calcification of A7R5 cells induced by hydroxyapatite nanoparticles. Mater. Sci. Eng. C Mater. Biol. Appl. 2020, 107, 110228. [CrossRef] [PubMed]

56. Zhao, Y.; Zhao, M.-M.; Cai, Y.; Zheng, M.-F.; Sun, W.-L.; Zhang, S.-Y.; Kong, W.; Gu, J.; Wang, X.; $\mathrm{Xu}$, M.-J. Mammalian target of rapamycin signaling inhibition ameliorates vascular calcification via Klotho upregulation. Kidney Int. 2015, 88, 711-721. [CrossRef]

57. He, H.-Q.; Law, B.Y.K.; Zhang, N.; Qiu, C.-L.; Qu, Y.-Q.; Wu, A.-G.; Han, Y.; Song, Q.; Zheng, W.-L.; Liu, Y.; et al. Bavachin Protects Human Aortic Smooth Muscle Cells Against beta-Glycerophosphate-Mediated Vascular Calcification and Apoptosis via Activation of mTOR-Dependent Autophagy and Suppression of beta-Catenin Signaling. Front. Pharm. 2019, 10, 1427. [CrossRef]

58. Chen, W.R.; Yang, J.Q.; Liu, F.; Shen, X.Q.; Zhou, Y.J. Melatonin attenuates vascular calcification by activating autophagy via an AMPK/mTOR/ULK1 signaling pathway. Exp. Cell Res. 2020, 389, 111883. [CrossRef]

59. Ma, W.-Q.; Sun, X.-J.; Wang, Y.; Zhu, Y.; Han, X.-Q.; Liu, N.-F. Restoring mitochondrial biogenesis with metformin attenuates beta-GP-induced phenotypic transformation of VSMCs into an osteogenic phenotype via inhibition of PDK4/oxidative stress-mediated apoptosis. Mol. Cell. Endocrinol. 2019, 479, 39-53. [CrossRef]

60. Chen, Y.; Zhang, L.S.; Ren, J.L.; Zhang, Y.R.; Wu, N.; Jia, M.Z.; Yu, Y.R.; Ning, Z.P.; Tang, C.S.; Qi, Y.F. Intermedin(1-53) attenuates aging-associated vascular calcification in rats by upregulating sirtuin 1. Aging 2020, 12, 5651-5674. [CrossRef]

61. Xu, M.; Liu, L.; Song, C.; Chen, W.; Gui, S. Ghrelin improves vascular autophagy in rats with vascular calcification. Life Sci. 2017, 179, 23-29. [CrossRef] [PubMed]

62. Wei, M.; Gan, L.; Liu, Z.; Liu, L.; Chang, J.R.; Yin, D.C.; Cao, H.L.; Su, X.L.; Smith, W.W. Mitochondrial-Derived Peptide MOTS-c Attenuates Vascular Calcification and Secondary Myocardial Remodeling via Adenosine Monophosphate-Activated Protein Kinase Signaling Pathway. Cardiorenal. Med. 2020, 10, 42-50. [CrossRef] [PubMed]

63. Ciceri, P.; Elli, F.; Cappelletti, L.; Tosi, D.; Braidotti, P.; Bulfamante, G.; Cozzolino, M. A new in vitro model to delay high phosphate-induced vascular calcification progression. Mol. Cell Biochem. 2015, 410, 197-206. [CrossRef] [PubMed] 
64. Liao, L.; Zhuang, X.; Li, W.; Su, Q.; Zhao, J.; Liu, Y. Polysaccharide from Fuzi protects against OxLDLinduced calcification of human vascular smooth muscle cells by increasing autophagic activity. Mol. Med. Rep. 2018, 17, 5109-5115. [CrossRef]

65. Yao, L.; Wang, J.; Tian, B.-Y.; Xu, T.-H.; Sheng, Z.-T. Activation of the Nrf2-ARE Signaling Pathway Prevents Hyperphosphatemia-Induced Vascular Calcification by Inducing Autophagy in Renal Vascular Smooth Muscle Cells. J. Cell. Biochem. 2017, 118, 4708-4715. [CrossRef] [PubMed]

66. Peng, Y.-Q.; Xiong, D.; Lin, X.; Cui, R.-R.; Xu, F.; Zhong, J.-Y.; Zhu, T.; Wu, F.; Mao, M.-Z.; Liao, X.-B.; et al. Oestrogen Inhibits Arterial Calcification by Promoting Autophagy. Sci. Rep-UK 2017, 7, 3549. [CrossRef] [PubMed]

67. Liu, D.; Cui, W.; Liu, B.; Hu, H.; Liu, J.; Xie, R.; Yang, X.; Gu, G.; Zhang, J.; Zheng, H. Atorvastatin protects vascular smooth muscle cells from TGF-beta1-stimulated calcification by inducing autophagy via suppression of the beta-catenin pathway. Cell. Physiol. Biochem. 2014, 33, 129-141. [CrossRef]

68. Xu, T.-H.; Qiu, X.-B.; Sheng, Z.-T.; Han, Y.-R.; Wang, J.; Tian, B.-Y.; Yao, L. Restoration of microRNA-30b expression alleviates vascular calcification through the mTOR signaling pathway and autophagy. J. Cell. Physiol. 2019, 234, 14306-14318. [CrossRef]

69. Zhang, X.; Chen, J.; Meng, Q.; Li, D.; Hu, F.-Z.; Zhu, Y.-Q.; Huang, Y.-Y.; Liu, Y.-N.; Sun, L.; Liang, Q.-H. The protective effects of long non-coding RNA-ANCR on arterial calcification. J. Bone Min. Metab. 2020, 38, 421-431. [CrossRef]

70. Shi, M.; Maique, J.; Shaffer, J.; Davidson, T.; Sebti, S.; Fernández, Á.F.; Zou, Z.; Yan, S.; Levine, B.; Moe, O.W.; et al. The tripartite interaction of phosphate, autophagy, and $\alpha$ Klotho in health maintenance. FASEB J. 2020, 34, 3129-3150. [CrossRef]

71. Ciceri, P.; Falleni, M.; Tosi, D.; Martinelli, C.; Cannizzo, S.; Marchetti, G.; D'Arminio Monforte, A.; Bulfamante, G.; Block, G.A.; Messa, P.; et al. Therapeutic Effect of Iron Citrate in Blocking Calcium Deposition in High Pi-Calcified VSMC: Role of Autophagy and Apoptosis. Int. J. Mol. Sci. 2019, 20, 5925. [CrossRef] [PubMed]

72. Yang, R.; Zhu, Y.; Wang, Y.; Ma, W.; Han, X.; Wang, X.; Liu, N. HIF-1alpha/PDK4/autophagy pathway protects against advanced glycation end-products induced vascular smooth muscle cell calcification. Biochem. Biophys. Res. Commun. 2019, 517, 470-476. [CrossRef] [PubMed]

73. Liu, Y.; Li, J.; Han, Y.; Chen, Y.; Liu, L.; Lang, J.; Yang, C.; Luo, H.; Ning, J. Advanced glycation end-products suppress autophagy by AMPK/mTOR signaling pathway to promote vascular calcification. Mol. Cell. Biochem. 2020, 471, 91-100. [CrossRef] [PubMed]

74. Chen, R.; Xu, Y.; Zhong, W.; Li, B.; Yang, P.; Wang, Z.Q.; Shao, C.; Wang, C.P.; Yan, J.C. Activation of CD137 Signaling Enhances Vascular Calcification through c-Jun N-Terminal Kinase-Dependent Disruption of Autophagic Flux. Mediat. Inflamm. 2018, 2018, 8407137. [CrossRef] [PubMed]

75. Xu, T.H.; Sheng, Z.; Li, Y.; Qiu, X.; Tian, B.; Yao, L. OGT knockdown counteracts high phosphate-induced vascular calcification in chronic kidney disease through autophagy activation by downregulating YAP. Life Sci. 2020, 261, 118121. [CrossRef]

76. Shiozaki, Y.; Miyazaki-Anzai, S.; Okamura, K.; Keenan, A.L.; Masuda, M.; Miyazaki, M. GPAT4-Generated Saturated LPAs Induce Lipotoxicity through Inhibition of Autophagy by Abnormal Formation of Omegasomes. iScience 2020, 23, 101105. [CrossRef]

77. Zhu, Y.; Han, X.-Q.; Sun, X.-J.; Yang, R.; Ma, W.-Q.; Liu, N.-F. Lactate accelerates vascular calcification through NR4A1-regulated mitochondrial fission and BNIP3-related mitophagy. Apoptosis Int. J. Program. Cell Death 2020, 25, 321-340. [CrossRef]

78. Zhu, Y.; Ji, J.-J.; Yang, R.; Han, X.-Q.; Sun, X.-J.; Ma, W.-Q.; Liu, N.-F. Lactate accelerates calcification in VSMCs through suppression of BNIP3-mediated mitophagy. Cell. Signal. 2019, 58, 53-64. [CrossRef]

79. Bhat, O.M.; Yuan, X.; Camus, S.; Salloum, F.N.; Li, P.L. Abnormal Lysosomal Positioning and Small Extracellular Vesicle Secretion in Arterial Stiffening and Calcification of Mice Lacking Mucolipin 1 Gene. Int. J. Mol. Sci. 2020, 21, 1713. [CrossRef]

80. Bhat, O.M.; Li, G.; Yuan, X.; Huang, D.; Gulbins, E.; Kukreja, R.C.; Li, P.L. Arterial Medial Calcification through Enhanced small Extracellular Vesicle Release in Smooth Muscle-Specific Asah1 Gene Knockout Mice. Sci. Rep. 2020, 10, 1645. [CrossRef] 
81. Bhat, O.M.; Yuan, X.; Cain, C.; Salloum, F.N.; Li, P.L. Medial calcification in the arterial wall of smooth muscle cell-specific Smpd1 transgenic mice: A ceramide-mediated vasculopathy. J. Cell. Mol. Med. 2020, 24, 539-553. [CrossRef] [PubMed]

82. Sudo, R.; Sato, F.; Azechi, T.; Wachi, H. 7-Ketocholesterol-induced lysosomal dysfunction exacerbates vascular smooth muscle cell calcification via oxidative stress. Genes Cells 2015, 20, 982-991. [CrossRef] [PubMed]

83. Mao, M.; Zhang, M.; Ge, A.; Ge, X.; Gu, R.; Zhang, C.; Fu, Y.; Gao, J.; Wang, X.; Liu, Y.; et al. Granzyme B deficiency promotes osteoblastic differentiation and calcification of vascular smooth muscle cells in hypoxic pulmonary hypertension. Cell Death Dis. 2018, 9, 221. [CrossRef] [PubMed]

84. Carracedo, M.; Persson, O.; Saliba-Gustafsson, P.; Artiach, G.; Ehrenborg, E.; Eriksson, P.; Franco-Cereceda, A.; Back, M. Upregulated Autophagy in Calcific Aortic Valve Stenosis Confers Protection of Valvular Interstitial Cells. Int. J. Mol. Sci. 2019, 20, 1486. [CrossRef]

85. Deng, X.-S.; Meng, X.; Venardos, N.; Song, R.; Yamanaka, K.; Fullerton, D.; Jaggers, J. Autophagy negatively regulates pro-osteogenic activity in human aortic valve interstitial cells. J. Surg. Res. 2017, 218, $285-291$. [CrossRef]

86. Somers, P.; Knaapen, M.; Kockx, M.; van Cauwelaert, P.; Bortier, H.; Mistiaen, W. Histological evaluation of autophagic cell death in calcified aortic valve stenosis. J. Heart Valve Dis. 2006, 15, 43-47; discussion 48.

87. Bonetti, A.; Della Mora, A.; Contin, M.; Gregoraci, G.; Tubaro, F.; Marchini, M.; Ortolani, F. Survival-Related Autophagic Activity Versus Procalcific Death in Cultured Aortic Valve Interstitial Cells Treated With Critical Normophosphatemic-Like Phosphate Concentrations. J. Histochem. Cytochem. 2017, 65, 125-138. [CrossRef]

88. Ambrosio, S.; Saccà, C.D.; Amente, S.; Paladino, S.; Lania, L.; Majello, B. Lysine-specific demethylase LSD1 regulates autophagy in neuroblastoma through SESN2-dependent pathway. Oncogene 2017, 36, 6701-6711. [CrossRef]

89. Chen, T.; Mao, H.; Chen, C.; Wu, L.; Wang, N.; Zhao, X.; Qian, J.; Xing, C. The Role and Mechanism of $\alpha$-Klotho in the Calcification of Rat Aortic Vascular Smooth Muscle Cells. Biomed. Res. Int. 2015, 2015, 194362. [CrossRef]

90. Dai, Q.; Xu, Z.; Ma, X.; Niu, N.; Zhou, S.; Xie, F.; Jiang, L.; Wang, J.; Zou, W. mTOR/Raptor signaling is critical for skeletogenesis in mice through the regulation of Runx2 expression. Cell Death Differ. 2017, 24, 1886-1899. [CrossRef]

91. Fitter, S.; Matthews, M.P.; Martin, S.K.; Xie, J.; Ooi, S.S.; Walkley, C.R.; Codrington, J.D.; Ruegg, M.A.; Hall, M.N.; Proud, C.G.; et al. mTORC1 Plays an Important Role in Skeletal Development by Controlling Preosteoblast Differentiation. Mol. Cell. Biol. 2017, 37. [CrossRef] [PubMed]

92. Sabatini, D.M. Twenty-five years of mTOR: Uncovering the link from nutrients to growth. Proc. Natl. Acad. Sci. USA 2017, 114, 11818-11825. [CrossRef] [PubMed]

93. Kim, H.; Kim, H.J.; Lee, K.; Kim, J.M.; Kim, H.S.; Kim, J.R.; Ha, C.M.; Choi, Y.K.; Lee, S.J.; Kim, J.Y.; et al. $\alpha$-Lipoic acid attenuates vascular calcification via reversal of mitochondrial function and restoration of Gas6/Axl/Akt survival pathway. J. Cell. Mol. Med. 2012, 16, 273-286. [CrossRef] [PubMed]

94. Frank, M.; Duvezin-Caubet, S.; Koob, S.; Occhipinti, A.; Jagasia, R.; Petcherski, A.; Ruonala, M.O.; Priault, M.; Salin, B.; Reichert, A.S. Mitophagy is triggered by mild oxidative stress in a mitochondrial fission dependent manner. Biochim. Biophys. Acta 2012, 1823, 2297-2310. [CrossRef]

95. Klionsky, D.J.; Abdelmohsen, K.; Abe, A.; Abedin, M.J.; Abeliovich, H.; Acevedo Arozena, A.; Adachi, H.; Adams, C.M.; Adams, P.D.; Adeli, K.; et al. Guidelines for the use and interpretation of assays for monitoring autophagy (3rd edition). Autophagy 2016, 12, 1-222. [CrossRef]

96. Scotto Rosato, A.; Montefusco, S.; Soldati, C.; Di Paola, S.; Capuozzo, A.; Monfregola, J.; Polishchuk, E.; Amabile, A.; Grimm, C.; Lombardo, A.; et al. TRPML1 links lysosomal calcium to autophagosome biogenesis through the activation of the CaMKK $\beta / V P S 34$ pathway. Nat. Commun. 2019, 10, 5630. [CrossRef]

97. Bakhshian Nik, A.; Hutcheson, J.D.; Aikawa, E. Extracellular Vesicles As Mediators of Cardiovascular Calcification. Front. Cardiovasc. Med. 2017, 4, 78. [CrossRef]

98. Zazzeroni, L.; Faggioli, G.; Pasquinelli, G. Mechanisms of Arterial Calcification: The Role of Matrix Vesicles. Eur. J. Vasc. Endovasc. Surg. 2018, 55, 425-432. [CrossRef]

99. Schurgers, L.J.; Akbulut, A.C.; Kaczor, D.M.; Halder, M.; Koenen, R.R.; Kramann, R. Initiation and Propagation of Vascular Calcification Is Regulated by a Concert of Platelet- and Smooth Muscle Cell-Derived Extracellular Vesicles. Front. Cardiovasc. Med. 2018, 5, 36. [CrossRef] 
100. Eitan, E.; Suire, C.; Zhang, S.; Mattson, M.P. Impact of lysosome status on extracellular vesicle content and release. Ageing Res. Rev. 2016, 32, 65-74. [CrossRef]

101. Li, G.; Huang, D.; Hong, J.; Bhat, O.M.; Yuan, X.; Li, P.L. Control of lysosomal TRPML1 channel activity and exosome release by acid ceramidase in mouse podocytes. Am. J. Physiol. Cell Physiol. 2019, 317, C481-C491. [CrossRef] [PubMed]

102. Teichgräber, V.; Ulrich, M.; Endlich, N.; Riethmüller, J.; Wilker, B.; De Oliveira-Munding, C.C.; van Heeckeren, A.M.; Barr, M.L.; von Kürthy, G.; Schmid, K.W.; et al. Ceramide accumulation mediates inflammation, cell death and infection susceptibility in cystic fibrosis. Nat. Med. 2008, 14, 382-391. [CrossRef] [PubMed]

103. Cuervo, A.M.; Wong, E. Chaperone-mediated autophagy: Roles in disease and aging. Cell Res. 2014, 24, 92-104. [CrossRef]

104. Leopold, J.A. Cellular mechanisms of aortic valve calcification. Circ. Cardiovasc. Interv. 2012, 5, 605-614. [CrossRef] [PubMed]

105. Meyer-Schwesinger, C. The ubiquitin-proteasome system in kidney physiology and disease. Nat. Rev. Nephrol. 2019, 15, 393-411. [CrossRef]

Publisher's Note: MDPI stays neutral with regard to jurisdictional claims in published maps and institutional affiliations.

(C) 2020 by the authors. Licensee MDPI, Basel, Switzerland. This article is an open access article distributed under the terms and conditions of the Creative Commons Attribution (CC BY) license (http://creativecommons.org/licenses/by/4.0/). 